\title{
Morphological and statistical features of reaction zones in MILD and premixed combustion
}

\author{
Y. Minamoto, N. Swaminathan, R. S. Cant, T. Leung \\ Department of Engineering, Cambridge University, Cambridge, CB2 1PZ, UK
}

\begin{abstract}
Direct numerical simulation (DNS) results of turbulent MILD premixed and conventional (undiluted) premixed combustion have been investigated to shed light on the physical aspects of reaction zones and their morphology in MILD combustion. Results of a premixed case are used for comparative analyses. The analyses show that the regions with strong chemical activity in MILD combustion are distributed over a substantial portion of the computational domain unlike in the premixed case where these regions are confined to a small portion of the domain. Also, interactions of reaction zones are observed in MILD combustion with their spatial extent increasing with dilution level. These interactions give an appearance of distributed combustion for MILD conditions. The morphology of these reaction zones is investigated using the Minkowski functionals and shapefinders commonly employed in cosmology. Predominant sheet-like structures are observed for the premixed combustion case whereas a pancake-like structure is observed as the most probable shape for the MILD cases. Spatial and statistical analyses of various fluxes involved in a progress variable transport equation are conducted to study autoignitive or propagative characteristics of MILD reaction zones. The results suggest that there are local regions with autoignition, propagating-flames,
\end{abstract}


and their coexistence for the conditions considered in this study. Typically, reaction dominated or ignition front and propagating-flame dominated regions are entangled for high dilution cases. Scalar gradient plays a strong role on whether reaction or propagating-flame dominated activities are favoured locally. Keywords: MILD combustion, flameless combustion, Direct numerical simulation (DNS), Minkowski functionals, autoignition, flame propagation

\section{Introduction}

Moderate and Intense Low-oxygen Dilution (MILD) combustion has the potential to improve combustion efficiency and reduce pollutant emissions simultaneously $[1,2,3]$. This type of combustion is associated with intense preheating and dilution, where (i) the elevated reactant temperature, $T_{r}$, is higher than the autoignition temperature, $T_{i g n}$, of a given fuel mixture and (ii) the temperature increase during combustion, $\Delta T=T_{p}-T_{r}$, is lower than $T_{i g n}$. The product temperature, $T_{p}$, is very low compared to that in conventional combustion even with the elevated reactant temperature because the reactant mixture is diluted with a large amount of exhaust gas so that the oxygen level in the reactant mixture is typically low, from 2 to $5 \%$ by volume [3]. MILD combustion is clearly distinguished from conventional premixed combustion since $T_{r}$ and $\Delta T$ are quite different in the two modes of combustion [3].

Combustion under MILD conditions has many advantages [1, 2, 3] as summarised briefly below. Firstly, combustion efficiency is enhanced by preheating the reactants using the heat recovered from the exhaust. Secondly, NO formation is suppressed significantly because of intense dilution of reactants resulting in low oxygen level and flame temperature. It typically takes a few seconds to 
produce substantial levels of thermal NO at around $1900 \mathrm{~K}$ but this time reduces to a few milliseconds at $2300 \mathrm{~K}[1,2]$. The maximum temperature in MILD combustion is typically less than $1900 \mathrm{~K}[1,3]$. Thirdly, combustion conditions having large $T_{r}$ and small $\Delta T$ help to suppress combustion noise and instabilities [1,2]. MILD combustion has been achieved in various configurations in previous studies $[1,2,3,4,5,6,7,8,9,10]$.

Previous studies employing direct photographs $[6,7,9]$ and laser thermometry $[4,5,7,11]$ suggest a uniform and distributed combustion under MILD conditions, often referred to as flameless combustion. On the contrary, the Probability Density Function (PDF) of temperature reported in [8] suggests the existence of thin reaction zones. Planar Laser Induced Fluorescence (PLIF) images of OH radicals also show fairly thin reaction zones $[4,5,7,11]$. A recent study using direct simulation data of MILD combustion of a methane-air mixture shows the existence of thin reaction zones which interact frequently leading to the appearance of distributed combustion [12]. Thus, it is imperative to analyse reaction zone characteristics to understand whether MILD reaction zones are thin or not since this understanding is important to develop appropriate modelling for turbulent MILD combustion.

Autoignition is also expected to play a role in MILD combustion because $T_{r}>T_{i g n}$ as noted in previous studies $[1,2,3]$. The role of autoignition on flame stabilisation has been investigated using the Jet in Hot Coflow (JHC) burner [5, $13,14,15]$ and in other configurations involving diluted and/or hot reactants and coflow [16, 17]. The PLIF images of $\mathrm{CH}_{2} \mathrm{O}$ in [5] suggest that the hot coflow initiates ignition of the MILD mixture after localised extinction is caused by the entrained cold surrounding air in the JHC configuration. However, PLIF images 
of $\mathrm{OH}[4,5,7,11]$ and temperature PDFs [8] suggest that the MILD combustion in the JHC burner involves propagating flames. Thus, an interplay between autoignition and propagating flame can be expected in MILD combustion [3, 18].

These contradicting views on MILD combustion involving either thin reaction zones or distributed combustion, and either autoignition or propagating flames, are the primary questions to be tackled in the present study. Direct numerical simulation (DNS) data are ideally suited for this purpose and a detailed analysis of MILD combustion DNS data is conducted to answer these questions. The morphology of turbulent MILD reaction zones is studied using the Minkowski functionals to be described in section 3.2. The autoignitive or propagative characteristics of these reaction zones are analysed using the balance of various fluxes in the transport equation of a reaction progress variable based on temperature.

This paper is organised as follows. The DNS methodology, construction of initial fields, and combustion conditions are briefly described in section 2 . The results are presented in section 3. The PDFs of reaction progress variable in MILD combustion are presented in section 3.1. The analysis using Minkowski functionals is discussed in section 3.2.1 and the flux-balance analysis is presented in section 3.3. The conclusions are summarised in the final section.

\section{DNS of MILD combustion and turbulent premixed flames}

Combustion of a lean methane-air mixture will be considered under both MILD and conventional premixed conditions. In this paper, MILD premixed combustion will be referred to as "MILD combustion" while conventional undiluted premixed combustion will be referred to as "premixed combustion". The conditions for the MILD combustion are created using the concept of exhaust gas recircu- 
lation (EGR) and thus the reactant mixture is made of unburnt and burnt gases distributed randomly in space and time. The construction of this field is described in section 2.2. Direct simulation of a complete EGR system is beyond the reach of current computational capability and thus a two-stage strategy is followed in this study. Before describing this strategy in detail in section 2.2, the numerical methods are discussed first.

\subsection{Numerical method and configuration}

The numerical code SENGA2 [19] employed for this study solves fully compressible transport equations for mass, momentum, total energy and mass fractions of various scalars involved in the combustion chemistry. The transport properties depend on temperature and a detailed chemical kinetics scheme can be used for combustion chemistry. This code has been used in several earlier studies on turbulent premixed flames $[20,21]$ involving one-step or complex chemistry. Here, a skeletal mechanism involving 16 species and 25 elementary reactions [22] is used. Binary Fickian diffusion is used with constant Lewis numbers for each species. The reference autoginition delay times computed using this diffusion model are comparable to those obtained using a multi-component diffusion model for MILD conditions [15]. Also, the use of multi-step chemistry and non-unity Lewis numbers is important for MILD combustion because of the role played by radicals and intermediate species present in the diluent. The values of burnt side temperature, laminar flame speed, thermal thickness, and reference autoignition delay times computed using the skeletal mechanism compared well with those obtained using GRI3.0 mechanism for the MILD conditions of this study.

The spatial derivatives are approximated on a uniform numerical grid using a tenth order central difference scheme which gradually reduces to a fourth or- 
der scheme near boundaries. The time integration is achieved using a third order Runge-Kutta scheme. The numerical stability of these schemes is maintained by using sufficiently small time steps $\left(\Delta t \leq 1 \times 10^{-8} \mathrm{~s}\right)$, which are dictated by the acoustic CFL condition. The computational domain is cubic, and inflow and non-reflecting outflow boundaries in the $x$ direction are specified using the Navier-Stokes Characteristic Boundary Condition [23] while periodic conditions are specified in the $y$ and $z$-directions. A similar computational domain with these boundary conditions is used for a conventional turbulent lean premixed flame, which is used for comparative analyses.

The mixture fields for MILD combustion contain pockets of exhaust and fresh gases. Such mixture fields are generated carefully in preprocessing steps to be dis-

cussed in section 2.2. These preprocessed fields of scalar mass fractions, $\hat{Y}_{i}[\underline{x}(t), y, z]$, velocity, $\hat{\boldsymbol{u}}[\underline{x}(t), y, z]$, and temperature, $\hat{T}[\underline{x}(t), y, z]$ are fed at an average velocity of $U_{i n}$ as inflowing fields, $Y_{i}(x=0, y, z, t), \boldsymbol{u}(x=0, y, z, t)$ and $T(x=0, y, z, t)$ through the inflow boundary located at $x=0$ of the computational domain. The symbol $\underline{x}(t)$ denotes the $x$ location of a scanning plane at time $t$ moving at a velocity of $U_{\text {in }}$ through the preprocessed fields. The method to construct these fields is described in the next subsection. For a DNS case of turbulent premixed flame, only the turbulent velocity field $\boldsymbol{u}$ is preprocessed. The species mass fractions and temperature at the inflow boundary are set to be constant values which correspond to the initial mixture composition.

\subsection{Preprocessing of initial and inflow mixture fields}

Direct numerical simulation of a complete MILD combustion system is not yet feasible because of the heavy computational cost involved. So, the simulation is split into two phases to mimic the physical processes of MILD combustion as 


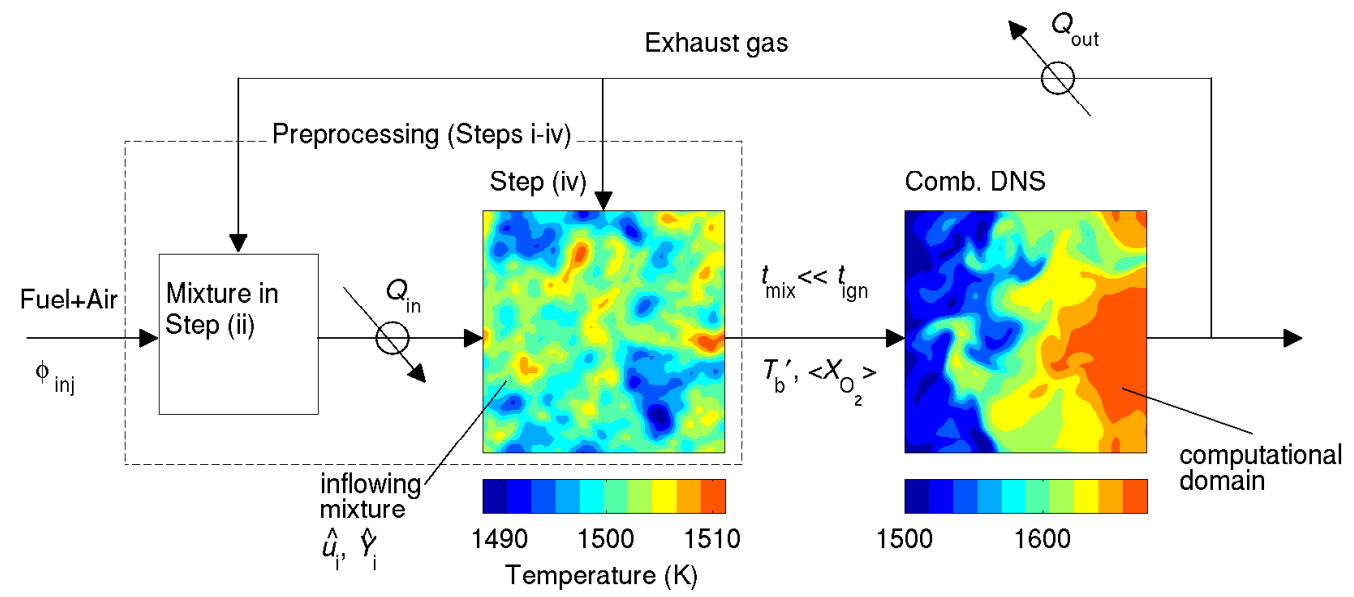

Figure 1. Schematics of the two-stage approach employed for the present MILD combustion DNS. Left box: mixing DNS domain. Right box: combustion DNS domain. Steps (i)-(iv) noted in the left box are described in section 2.2

noted in the previous subsection. The first phase involves preprocessing of a nonuniform and inhomogeneous mixture field which is consistent with the required turbulence and combustion conditions. The second phase is the turbulent MILD combustion in which the inhomogeneous mixture field generated in the first phase is used. These two phases are represented pictorially in Fig. 1. Here, the symbol $\phi_{\text {inj }}$ is the equivalence ratio and $Q$ represents cooling and heating of the product and reactant mixtures respectively. The desired mixture fields of $\hat{\boldsymbol{u}}, \hat{Y}_{i}$ and $\hat{T}$ are obtained following the steps in $[12,24]$, which are described briefly below.

A fully developed homogeneous isotropic turbulence field is obtained first by simulating a freely decaying non-reacting turbulence field evolving from a specified initial spectrum [25]. Then, a homogeneous scalar field is obtained by specifying a scalar energy spectrum as in [26], which is taken as the initial field of reaction progress variable $c_{Y}$ defined as $c_{Y}=1-Y_{f} / Y_{f, r}$, varying from 0 to 1 , where $Y_{f}$ is the fuel mass fraction and the subscript $r$ denotes the reactant mixture. 
The species mass fractions obtained from a one-dimensional freely propagating laminar flame with desired MILD conditions are then mapped on to the $c_{Y}$ field. The laminar flame is computed using the same chemical kinetics mechanism used for the DNS. The unburnt mixture of this laminar flame is diluted with products of fully burnt mixture $\left(X_{\mathrm{H}_{2} \mathrm{O}}: X_{\mathrm{CO}_{2}}=2: 1\right)$ so that the $\mathrm{O}_{2}$ mole fraction in the reactant mixture $X_{\mathrm{O}_{2}, r}$ matches a desired level. The initial temperature is set to a constant value, $T_{m}$, to be specified later. Finally, these scalar and velocity fields are allowed to evolve for about one large eddy turnover time in a periodic domain without any chemical reaction to mimic EGR-mixing for a duration much shorter than the reference autoignition delay time, $\tau_{i g n}$, for the chosen mixture conditions. The internal energy equation is also solved during this mixing process, which creates a maximum temperature fluctuation of about $2 \%$ of the mean value, $T_{m}$. A sample field is shown on the left in Fig. 1. The mean and variance of the $c_{Y}$ field at the end of this EGR mixing process are respectively $\left\langle c_{Y}\right\rangle \approx 0.50$ and $\left\langle c_{Y}^{\prime 2}\right\rangle \approx 0.09$ for all the MILD cases considered for this study. More elaborate discussion on the construction of the initial fields can be found in $[12,24]$

Bilger's mixture fraction [27] has a variation of about $\pm 5 \%$ of the mean value, $\langle\xi\rangle$, in the initial field. The equivalence ratio obtained using $\phi=\left(1-\xi_{s t}\right) \xi /(1-$ $\xi) \xi_{s t}$, where $\xi_{s t}$ is the stoichiometric mixture fraction, gives a mean value of $\langle\phi\rangle=$ 0.8 for all the cases considered in this study. The calculation of $\xi$ is based on the boundary condition for the air stream diluted with products to a desired level of oxygen and a pure fuel stream as shown in Fig. 1.

A typical $c_{Y}$ field generated at the end of the EGR mixing process is shown in Fig. 2. The contours depicted for a typical $x-y$ plane shows that the field is not only inhomogeneous but also turbulent. The PDF of $c_{Y}$ constructed from the initial 


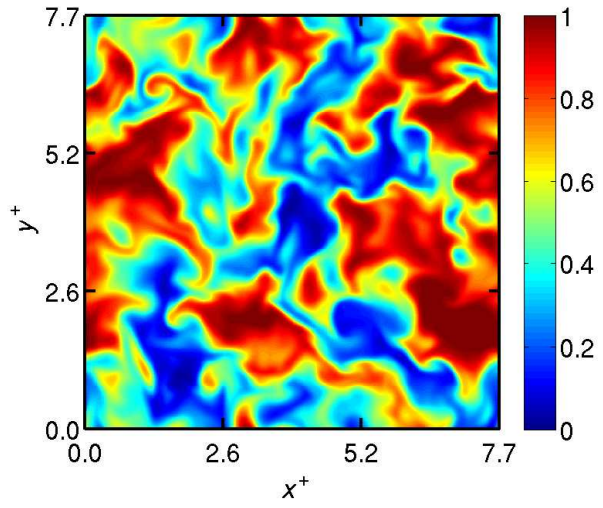

(a)

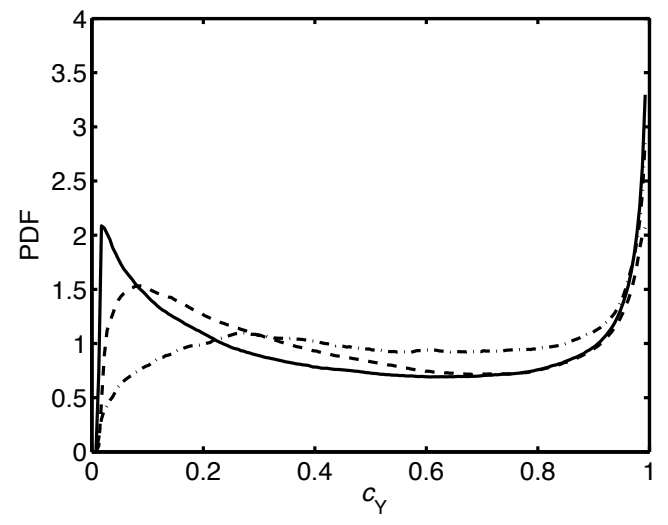

(b)

Figure 2. (a) Spatial variation of $c_{Y}$ field at mid $x-y$ plane preprocessed using the steps explained in Sec. 2.2 for Case B1. (b) PDF of $c_{Y}$ in the preprocessed field. Solid line: preprocessed field to be used for Case A1, dashed line: for Case A2, and dash-dotted line: for Case B1. Conditions of these cases are explained in Sec. 2.3.

field is also shown in Fig. 2. The turbulent mixing and molecular diffusion that have occurred during the EGR mixing process yield samples with intermediate values, $0<c_{Y}<1$. The PDF of $c_{Y}$ shows that a substantial portion of mixture is well mixed after this procedure, while there are local mixtures which still have either fresh or exhaust gases. The PDF is shown for three MILD cases considered in this study and the thermo-chemical and turbulence conditions of these cases are discussed next.

\subsection{Combustion conditions}

The thermochemical conditions of the laminar flames used in the generation of the initial and inflowing fields described in section 2.2 are given in Table 1 . The flames A and B have diluted reactant mixture as signified by the mole frac- 
Table 1. Summary of laminar flame properties. Units are Kelvin (K), metre (m) and second (s).

\begin{tabular}{llcccccccc}
\hline & $X_{\mathrm{CH}_{4}, r}$ & $X_{\mathrm{O}_{2}, r}$ & $X_{\mathrm{H}_{2} \mathrm{O}, r}$ & $X_{\mathrm{CO}_{2}, r}$ & $T_{r}$ & $T_{p}$ & $S_{L}$ & $10^{3} \delta_{\text {th }}$ & $10^{3} \tau_{\text {ign }}$ \\
\hline Flame A & 0.019 & 0.048 & 0.121 & 0.061 & 1500 & 1865 & 3.20 & 0.69 & 4.97 \\
Flame B & 0.014 & 0.035 & 0.132 & 0.066 & 1500 & 1775 & 2.15 & 0.94 & 6.71 \\
Flame C & 0.078 & 0.194 & 0.0 & 0.0 & 600 & 2179 & 1.18 & 0.37 & - \\
\hline MIFE A & 0.0095 & 0.035 & 0.136 & 0.064 & 1500 & 1692 & 2.62 & 1.00 & 5.42 \\
MIFE B & 0.0060 & 0.025 & 0.143 & 0.068 & 1500 & 1624 & 1.66 & 1.29 & 6.96 \\
\hline
\end{tabular}

tion, $X_{i}, r$, of species $i$ in the reactant mixture and thus these two laminar flames are used in the construction of mixture fields for the turbulent MILD cases. The burning velocity of these laminar flames is given in Table 1 as $S_{L}$ and the thermal thickness is defined as $\delta_{t h}=\left(T_{p}-T_{r}\right) /|\nabla T|_{\max }$. The reference autoignition delay time for these mixtures is also given in the table. Here, this autoignition delay times are computed using a zero-dimensional, constant pressure homogeneous reactor model and employing the maximum temperature gradient criterion. Flame $\mathrm{C}$ is a classical premixed flame, which is indicated by the large temperature rise, $T_{p}-T_{r}$, and the reactant mole fractions listed in Table 1 .

The temperature rise across the laminar flames with the diluted and preheated mixtures is observed to be relatively small, about $370 \mathrm{~K}$ for Flame A and $280 \mathrm{~K}$ for Flame B, which is typical of MILD combustion. Spatial variations of species mass fraction in the inflowing mixture fields are inevitable, as seen in Fig. 2a, when the MILD combustion occurs in three dimensions and it is not possible to represent this spatial variation in a representative 1D laminar flame. Indeed, the effect of additional dilution resulting from spatial variation reduces the burnt mixture temperature in the turbulent cases by about $150 \mathrm{~K}$ compared to the respective laminar flames [12]. To account for the dilution effect even for laminar flames, 
Table 2. turbulent combustion conditions.

\begin{tabular}{c||ccccccccccc}
\hline & $X_{\mathrm{O}_{2}, r}^{\max }$ & $\left\langle X_{\mathrm{O}_{2}, r}\right\rangle$ & $\langle\xi\rangle$ & $\xi_{s t}$ & $U_{i n} / S_{L}$ & $u^{\prime} / S_{L}$ & $l_{0} / \delta_{F}$ & $l_{0} / \delta_{t h}$ & $\mathrm{Re}_{l_{0}}$ & $\mathrm{Da}$ & $\mathrm{Ka}$ \\
\hline Case A1 & 0.048 & 0.035 & 0.011 & 0.014 & 9.6 & 6.26 & 10.8 & 1.48 & 96.2 & 1.72 & 4.78 \\
Case A2 & 0.048 & 0.035 & 0.011 & 0.014 & 9.6 & 3.80 & 12.3 & 1.70 & 67.0 & 3.25 & 2.11 \\
Case B1 & 0.035 & 0.025 & 0.008 & 0.010 & 15.1 & 9.88 & 6.8 & 1.15 & 96.1 & 0.69 & 11.9 \\
Case C & 0.194 & 0.194 & 0.044 & 0.055 & 3.0 & 2.19 & 12.3 & 2.11 & 38.5 & 5.64 & 0.92 \\
\hline
\end{tabular}

the mass fractions of major species in the reactant mixture are computed based on the volume average of the respective species in the preprocessed DNS initial field obtained after the EGR mixing step. A laminar flame having this reactant mixture is named as a MILD Flame Element (MIFE) in this study [24]. As one would expect, this averaging yields significantly smaller mole fraction values as noted in Table 1 for MIFEs A and B compared to the Flames A and B. Also, the burnt mixture temperature, $T_{p}$, is only about $27 \mathrm{~K}$ higher than the DNS values (this is the maximum difference observed). For these reasons, MIFEs are taken to be representative flamelets for the MILD combustion cases investigated in this study and their thermochemical attributes listed in Table 1 are used to normalise the respective DNS results. For example, length, gradient of reaction progress variable and reaction rate are respectively normalised using $\delta_{t h}, 1 / \delta_{t h}$ and $\rho_{r} S_{L} / \delta_{t h}$. The normalised quantities are denoted using a superscript "+".

\subsubsection{Conditions for turbulent MILD combustion}

Three MILD and one classical premixed combustion case were simulated. The turbulence and thermochemical conditions of these four cases are given in Table 2 . The thermochemical conditions for Cases A1 and A2 are based on Flame A. Thus, they have the same dilution level, but their turbulence conditions are different as shown in Table 2. Case A1 has the same turbulence field as in Case B1, but 
uses the same thermochemical conditions as Case A2. All of the four cases have an equivalence ratio of $\phi=0.8$ and the autoignition temperature for this lean methane-air mixture is $1100 \mathrm{~K}$. The temperature of the initial and inflowing mixture is set to be $T_{m} \approx 1500 \mathrm{~K}$ for the MILD combustion cases, and $600 \mathrm{~K}$ for the premixed combustion Case $\mathrm{C}$. The high value of $T_{m}$ for the MILD combustion cases is comparable to that used in experiments [28]. This inlet temperature together with the low mole fraction of $\mathrm{O}_{2}$ suggests that the combustion conditions are strictly in the MILD regime.

The maximum $X_{\mathrm{O}_{2}, r}^{\max }$ and averaged $\left\langle X_{\mathrm{O}_{2}, r}\right\rangle$ mole fractions of oxygen in the reactant mixture for the MILD cases are significantly smaller than for Case C because of the high dilution levels (see Table 2). These dilution levels are comparable to previous studies $[2,6,3]$.

The values of mean $\langle\xi\rangle$ and stoichiometric $\xi_{s t}$ mixture fractions are also given in Table 2. The RMS of velocity fluctuations and the integral length scale of the initial turbulence field are denoted respectively as $u^{\prime}$ and $l_{0}$ in Table 2 . The Zeldovich thickness is $\delta_{F}=\left(\lambda / \rho c_{p}\right) / S_{L}$, where $\lambda$ and $c_{p}$ are respectively the mixture thermal conductivity and specific heat capacity at constant pressure. The Reynolds number, $\operatorname{Re}_{l_{0}}=u^{\prime} l_{0} / \nu_{r}$, with $\nu_{r}$ as the kinematic viscosity of reactant mixture, is varied respectively from 67 to 96 for the MILD cases. An estimate of the Reynolds number for MILD combustion in previous studies [29, 11, 13] using the information for reacting jet flows $[30,31,32,33]$ suggests that the values of $\operatorname{Re}_{l_{0}}$ in Table 2 are comparable to those for previous MILD combustion experiments. The turbulence level for Case $\mathrm{C}$, the premixed combustion case, is deliberately set to be a small value to help to contrast the behaviour of reaction zones and their structure in MILD conditions and in premixed combustion with 
large Damköhler number. The Damköhler and Karlovitz numbers are defined as $\mathrm{Da}=\left(l_{0} / \delta_{F}\right) /\left(u^{\prime} / S_{L}\right)$ and $\mathrm{Ka} \approx\left(u^{\prime} / S_{L}\right)^{3 / 2}\left(l_{0} / \delta_{F}\right)^{-1 / 2}$. The three MILD cases are in the thin-reaction zones regime, and Case $\mathrm{C}$ is near the border between the thin-reaction zones and corrugated flamelets regimes in a turbulent combustion regime diagram [34].

\subsubsection{Computational detail}

The computational domain is of size $L_{x} \times L_{y} \times L_{z}=10.0 \times 10.0 \times 10.0 \mathrm{~mm}^{3}$ for the three MILD cases and $L_{x} \times L_{y} \times L_{z}=10.0 \times 5.0 \times 5.0 \mathrm{~mm}^{3}$ for the premixed flame, Case C. If the computational domain is normalised using $\delta_{t h}$ of the respective laminar flames (MIFEs for MILD and Flame $\mathrm{C}$ for premixed cases), $L_{x}^{+} \times L_{y}^{+} \times L_{z}^{+}=10^{3}$ for Cases A1 and A2, $(7.75)^{3}$ for Case B1, and $26.7 \times 13.4 \times 13.4$ for Case $\mathrm{C}$. These domains are discretised using $512 \times 512 \times 512$ mesh points for Cases A1 and A2, $384 \times 384 \times 384$ mesh points for Case B1, and $512 \times 256 \times 256$ mesh points for Case C. These meshes ensure that there are at least 15 mesh points inside the respective thermal thickness $\delta_{t h}$.

The simulations of the MILD cases were run for 1.5 flow-through times to ensure that the initial transients had left the domain before collecting data for statistical analysis. The flow-through time is defined as $\tau_{D}=L_{x} / U_{i n}$, which is the mean convection time from the inflow to the outflow boundary. The simulations were then continued for one additional flow-through time and 80 data sets were collected. For Case C, 93 data sets were collected over a time of $0.56 \tau_{D}$ after allowing one flow-through time for initial transients to exit the computational domain. These simulations have been run on Cray XE6 systems using 4096 cores with a wall-clock time of about 120 hours for Case A1 and Case A2, which have the largest number of mesh points, and using 16384 cores with 80 hours of wall- 
clock time for Case C.

\section{Results and Discussion}

\subsection{Temperature and reaction zone behaviour}

Typical PDFs of normalised temperature, $c_{T}=\left(T-T_{r}\right) /\left(T_{p}-T_{r}\right)$, are shown in Fig. 3 for various $x^{+}$locations for the Case B1 in Table 2. These PDFs are constructed using samples collected over the entire sampling period. This PDF indicating a bimodal behaviour for the premixed case is also shown for $x^{+}=17.5$ in Fig. 3. The PDFs of the MILD case show neither a sharp peak nor a bimodal behaviour. The PDF for the $x^{+}=0.30$ location, which is near the inlet boundary, shows that unburnt gases are predominant at this region. The PDF shows a plateau in $0.2 \leq c_{T} \leq 0.7$ for the location $x^{+}=2.9$ suggesting distributed combustion. As one moves further downstream, the probability of finding burnt gases increases and the PDF distribution is relatively broader compared to that of the premixed case.

Typical contours of $c_{T}$ and $\hat{\omega}_{c_{T}}$, which is the normalised reaction rate of $c_{T}$, are shown in Fig. 4 for a $x-y$ plane located at $z=L_{z} / 2$. The reaction rate is calculated as $\omega_{c_{T}}=\dot{Q} / c_{p}\left(T_{p}-T_{r}\right)$, where $\dot{Q}$ is the heat release rate. The contours of $\hat{\omega}_{c_{T}}=\omega_{c_{T}} / \omega_{c_{T} \text {, max }}=0.2$ and 0.5 shown in Fig. 3 for the premixed case form thin reaction zones with a typical thickness of about $\delta_{t h}$, where $\omega_{c_{T} \text {, max }}$ is the global maximum reaction rate at $t=1.5 \tau_{D}$. This behaviour is consistent with the bimodal PDF shown in Fig. 3 for the premixed case. Furthermore, the peak reaction rate is located along $c_{T}=0.6$ contour (grey dashed line), which is consistent with a previous observation [35] on the relation between $c_{T}$ and $\omega_{c_{T}}$. Although the value of Da for Case C is larger than for the MILD cases (see Table 2), previ- 


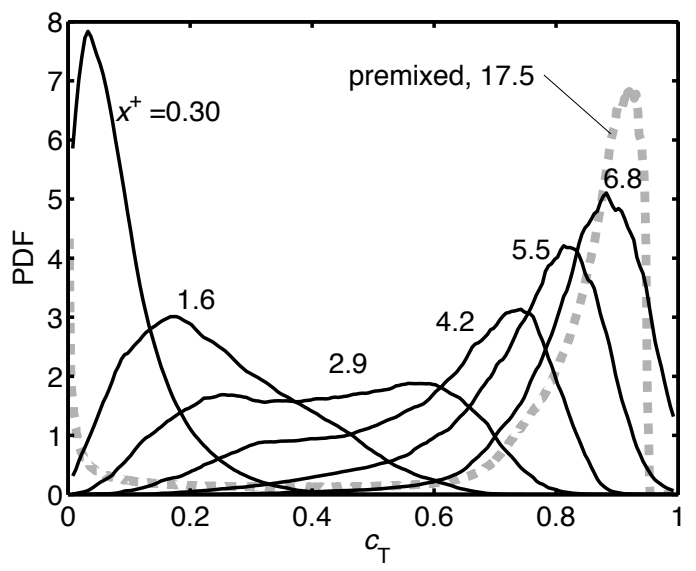

Figure 3. PDFs of $c_{T}$ for various $x^{+}$locations for Case B1. A typical PDF of the premixed case is also shown for $x^{+}=17.5$.

ous DNS $[36,37,38]$ of turbulent premixed flames with Da $<0.69$ showed thin sheet-like reaction zones with some interactions.

For the MILD cases, regions with $\hat{\omega}_{c_{T}} \geq 0.2$ occupy a substantial part of the combustion domain and the extent of such regions increases with dilution and turbulence levels. Thin reaction zones are also observed locally, which are typically surrounded by the $\hat{\omega}_{c_{T}} \geq 0.5$ contours. Such regions do not seem to follow a particular $c_{T}$ contour as for the premixed case. Also these regions are spread out in the domain to form a patchy appearance. Such patchy reaction zones are also observed in OH PLIF images of a previous experimental study [8]. Despite the existence of such thin local reaction zones, the temperature field is distributed as shown in the PDF of $c_{T}$ in Fig. 3. This results from broader and distributed reaction zones, which are also seen in Fig. 4. The patchy appearance or distributed nature of the reaction zones results from their abundant and frequent interactions. Previous analysis of time histories of the reaction zones has identified these in- 
teractions [12], which are not considered further in this study. Also, the intense reaction zones near the inlet boundary observed in Fig. 4 for the MILD cases are due to the radicals and intermediate species present in the reactant mixture diluted using products. The presence of these species and the high temperature of the reactant mixture will significantly shorten the autoignition delay time.

The reaction zones represented by $\hat{\omega}_{c_{T}} \geq 0.5$ in Fig. 4 are analysed next to study their morphological characteristics in order to understand whether these reaction zones are thin or not.

\subsection{Morphology of reaction zones}

\subsubsection{Minkowski functional and shapefinders}

The reaction zone morphology is analysed using Minkowski functionals [39], which help to define shapefinders. The shapefinders are characteristic scales for length, $L$, width, $W$, and thickness, $T$, of a three dimensional object of interest. The Minkowski functionals and shapefinders have been used to quantify isodensity structures in cosmology [40, 41, 42, 43, 44, 45, 46, 47, 48], magnetic field structures in magneto-hydrodynamics [49] and structures of homogeneous turbulence [50]. There are $(n+1)$ Minkowski functionals for a $n$-dimensional object and the 4 functionals for a 3D object are given as $[43,48]$

$$
\begin{gathered}
V_{0}=\mathcal{V}, \\
V_{1}=\frac{\mathcal{S}}{6}, \\
V_{2}=\frac{1}{3 \pi} \int_{S} \frac{\kappa_{1}+\kappa_{2}}{2} d S,
\end{gathered}
$$




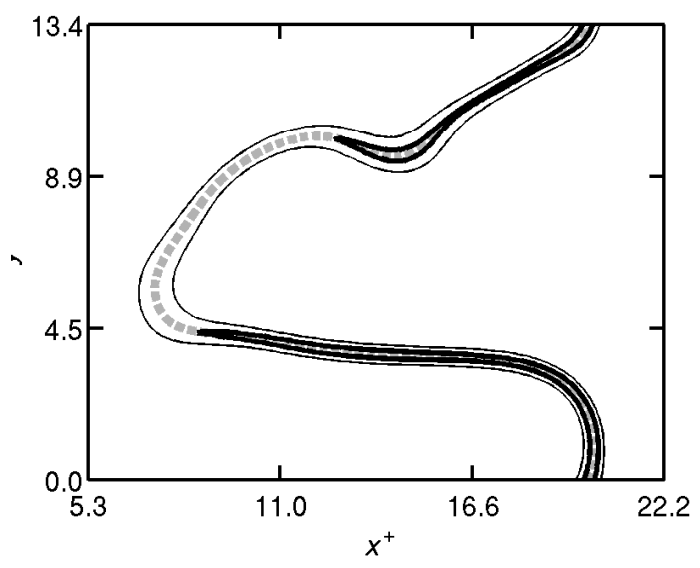

(a)

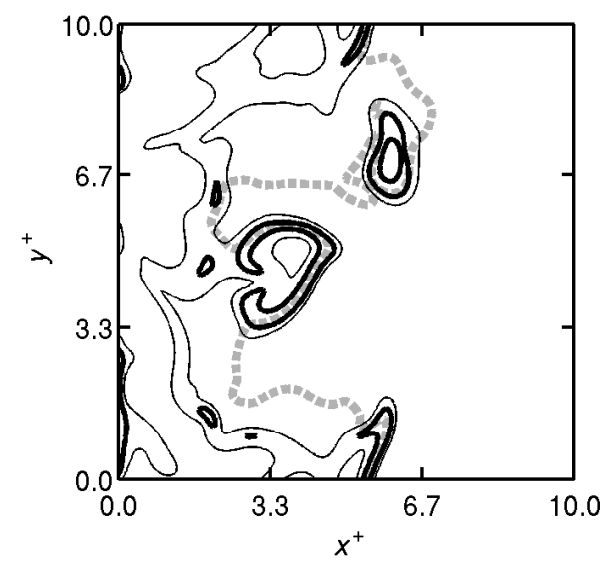

(c)

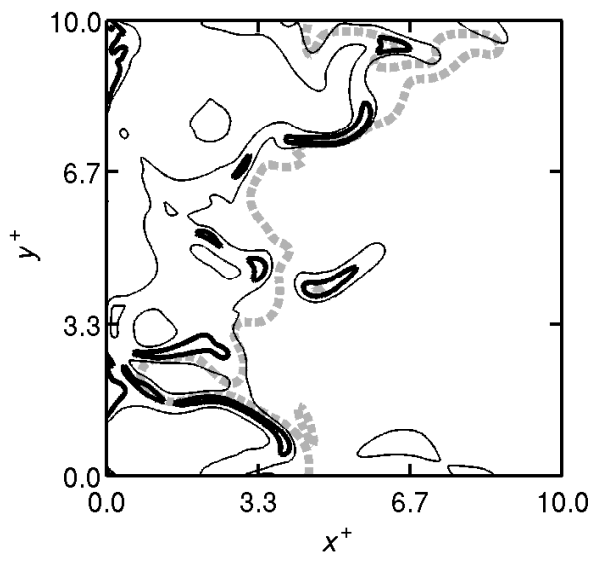

(b)

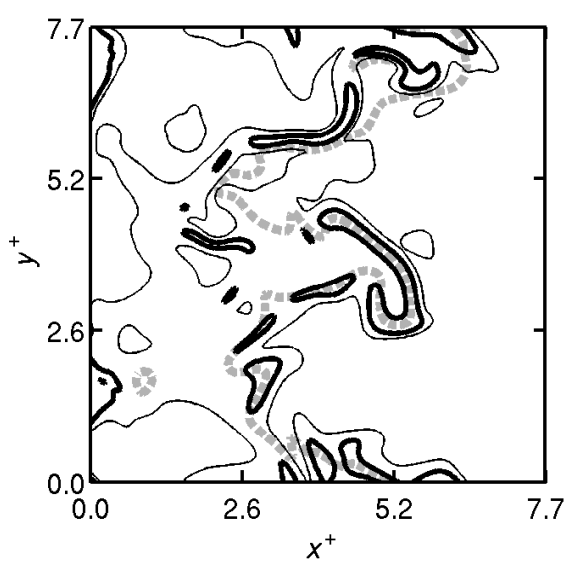

(d)

Figure 4. Contours of $c_{T}$ and $\hat{\omega}_{c_{T}}$ in the mid $x$ - $y$ planes for (a) Case C, (b) Case A1, (c) Case A2, and (d) Case B1. Thin solid line is $\hat{\omega}_{c_{T}}=0.2$, thick solid line is $\hat{\omega}_{c_{T}}=0.5$, and thick grey dashed line is $c_{T}=0.6$. Note that only $5.3 \leq x^{+} \leq 22.2$ of the computational domain is shown for Case C. All snapshots are taken at $t=1.5 \tau_{D}$. 


$$
V_{3}=\frac{1}{2 \pi} \int_{S}\left(\kappa_{1} \kappa_{2}\right) d S
$$

where $\mathcal{V}$ is the volume of the object enclosed by its surface, $S$, with a surface area of $\mathcal{S}$, and $\kappa_{1}$ and $\kappa_{2}\left(\kappa_{1} \geq \kappa_{2}\right)$ are the two principal curvatures at a given point on $S$. It is apparent that the mean and Gaussian curvatures integrated over the entire surface are involved respectively in $V_{2}$ and $V_{3}$, and local values of these curvatures are used in many turbulent combustion models. The integrated Gaussian curvature, $V_{3}$, is the Euler characteristic, $\chi$, which is a topological invariant of the three-dimensional object of interest. These four functionals defined in Eqs. (1) to (4) are Galilean invariant morphological properties of a three dimensional object. In the present study, the Minkowski functionals are computed for many objects identified using iso-surfaces of reaction rate and extracted by employing a grid cell counting method $[42,44]$.

Three characteristic scales for the shapefinder are obtained from the four functionals given in Eqs. (1) to (4) as [43]:

$$
\begin{gathered}
\text { Thickness, } T \equiv \frac{V_{0}}{2 V_{1}}, \\
\text { Width, } W \equiv \frac{2 V_{1}}{\pi V_{2}}, \\
\text { Length, } L \equiv \frac{3 V_{2}}{2 V_{3}},
\end{gathered}
$$

and they satisfy $L \geq W \geq T$ for any convex body [44]. Also this inequality holds for closed partially concave objects since the Minkowski functionals are additive $[44,50]$. These four functionals have to be positive to obtain physically meaningful shapefinders using Eqs. (5) to (7). However, $V_{3}=\chi$ can be positive or 
negative or zero because it is related to the three-dimensional genus $G=1-0.5 \chi$, which is defined as the number of cuts that can be made along a simple curve on an object without splitting it. For an object made by a single closed surface $G=0$ and thus $V_{3}=2$. If there is a hole on the object, say for example a torus, then $G=1$ and thus $V_{3}=0$. For a pretzel $G=2$ and thus $V_{3}=-2$. Hence, $V_{3}$ is related to the number of holes, $N_{\text {hole }}$, on the object as $V_{3} \approx 1-N_{\text {hole }}$. This creates a problem for the definition of $L$ in Eq. (7) because $L$ is singular for $V_{3}=0$ and it becomes unphysical when $V_{3}<0$. To avoid this, an alternative definition of $L$ is written for objects with non-zero $G$ or $V_{3} \leq 0$ as [45]:

$$
L \equiv \frac{3 V_{2}}{4(G+1)}
$$

The definition of $L$ in Eqs. 7 and 8 is identical for objects with no holes, $G=0$. For objects with holes, $G>0, L$ signifies a characteristic length between holes and it implies a representative radius of a circular torus when $G=1$ [50]. For a sphere of radius $r$, Eqs. (1) to (8) give $T=W=L=r$. For an infinitely long cylinder with radius $r_{1}$, these length scales are $(T, W, L)=\left(1.5 r_{1}, 2 r_{1}, \infty\right)$ and for an infinitely large thin circular disk of thickness $\delta$, they are $(T, W, L)=$ $(1.5 \delta, \infty, \infty)$. Thus, these three quantities yield representative scales for the spatial extent of a structure and do not give its exact dimensions except for a sphere.

Dimensionless shapefinders are defined as [43]:

$$
\begin{gathered}
\text { Planarity, } P \equiv \frac{W-T}{W+T}, \\
\text { Filamentarity, } F \equiv \frac{L-W}{L+W} .
\end{gathered}
$$




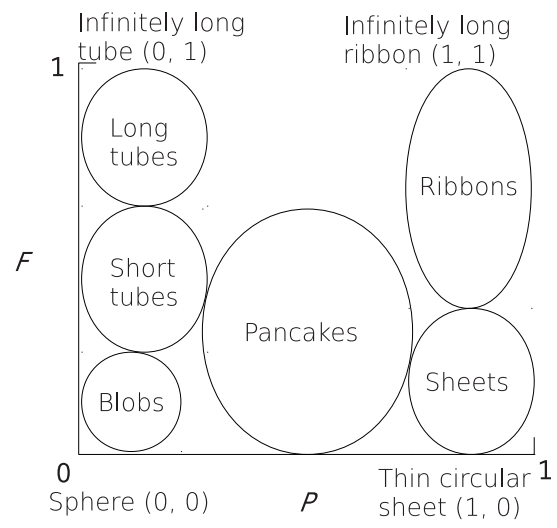

Figure 5. A representative map of shapefinders [50].

These two quantities allow a convenient visual representation of the geometry for a group of objects in a two-dimensional $(P, F)$ space. They vary from 0 to 1 when the inequality $L \geq W \geq T$ is met. The filamentarity approaches to 1 when $L$ is very large compared to $W$ and $T$. The planarity is close to 1 when $W \gg T$. Thus a long tube has $F \sim 1$ and $P \sim 0$ while a sheet has $F \sim 0$ and $P \sim 1$. Typical shapes are marked in the shapefinder, $(P, F)$, map shown in Fig. 5. It is obvious that $(P, F)=(0,0)$ for a spherical object.

\subsubsection{Shape of reaction zones}

The reaction zones are identified first using a threshold for $\omega_{c_{T}}^{+}$and this identification introduces some subjectivity in the analysis depending on the threshold value used. Also, the use of a single threshold value for all the cases does not identify regions with similar reaction rate magnitudes since the normalising factor $\rho_{r} s_{L} / \delta_{t h}$ is not the same for all the cases considered in this study. To remove these ambiguities, the threshold value is chosen using $\mathcal{Z}(\xi)=\left\langle\Delta S N_{c} \omega_{c_{T}} \mid \omega_{c_{T}}=\xi\right\rangle$ rather than just $\omega_{c_{T}}$, where $N_{c}=D_{c}\left(\nabla c_{T} \cdot \nabla c_{T}\right)$ is the scalar dissipation rate 
and $\Delta S$ is the surface area identified using $\omega_{c_{T}}=\xi$. The scalar dissipation rate and reaction rate are large when the reaction zones have propagative characteristics (rather they are propagating flames). Thus, a large value of $\mathcal{Z}$ automatically implies propagating flames and so their morphological characteristics can be compared meaningfully to those for premixed flames. Furthermore, using only a threshold value for $\omega_{c_{T}}^{+}$does not allow us to differentiate between the reaction and propagating-flame dominated structures.

The variations of $\mathcal{Z}(\xi) / \mathcal{Z}_{\max }$ with $\xi^{+}$for all the cases are shown in Fig. 6 and the value of $\xi_{1}^{+}$corresponding to $\mathcal{Z}(\xi) / \mathcal{Z}_{\max }=1$ in each case is chosen as the threshold to identify reaction zone iso-surfaces for morphological analysis. It is found that these surfaces enclose about the top $50 \%$ of the reaction rate in each case and thus they represent regions with intense reaction. It has also been verified that the statistics of shapefinders do not change unduly when the threshold is set to a value $20 \%$ smaller than $\xi_{1}^{+}$. Furthermore, the use of $c_{Y}$ and its reaction rate does not change the conclusions from this analysis. There were unclosed objects because of their interaction with inflow/outflow boundaries and these objects were excluded from further consideration since the Minskowski functionals require closed objects.

For most of the objects identified using the threshold value of $\xi_{1}^{+}$as noted above, the surface average of $\kappa_{1} \kappa_{2}$ is positive for all the four cases considered in this study. This implies that $V_{3}$ is also positive for these objects. However, the joint PDFs of $\kappa_{1}$ and $\kappa_{2}$ (not shown here) for the identified structures suggest that there are some negative principal curvature(s) locally. Thus, these structures have local concavity possibly with holes, although they are made of convex surface in an integral sense. Therefore, Eq. (8) is used to compute $L$ in the present analysis. 


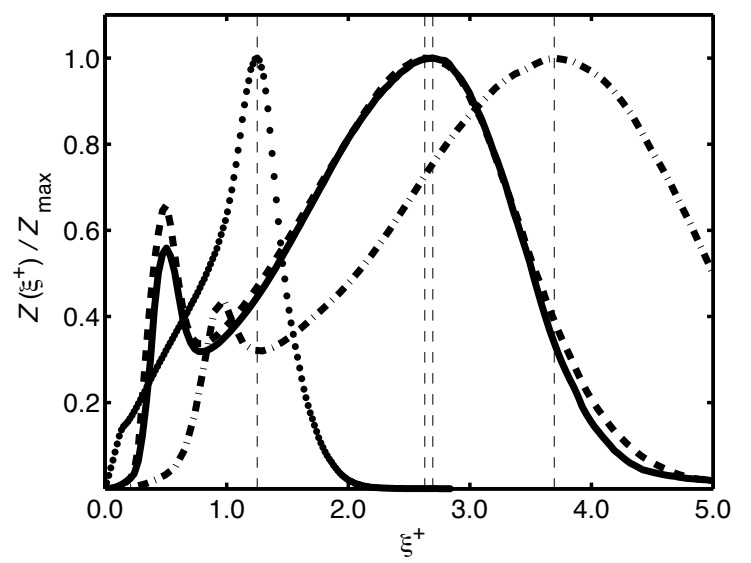

Figure 6. Variation of $\mathcal{Z}(\xi) / \mathcal{Z}_{\max }$ with $\xi^{+}$in Case A1 (solid line), Case A2 (dashed), Case B1 (dash-dotted) and Case C (dotted).

Also, a small fraction (about 15\%) of these structures have a multiply connected surface because of the presence of holes resulting from reaction zone contortions and their interaction due to turbulence.

The objects extracted using the post processing steps discussed above are analysed further to understand if the MILD combustion involves flame sheets, thin or distributed reaction zones. Two of these objects chosen arbitrarily are shown in Fig. 7 as examples and these are obtained from the MILD cases A1, Fig. 7a, and A2, Fig. 7b. As one sees, these objects are made of multiply connected surface and it is not easy to say if they can be classified as thin sheets or thin reaction zones based on a visual examination without any subjectivity. The Minkowski functionals and shapefinders discussed in the previous subsection help us on this matter as one shall see in the following discussion.

The values of these functionals, three characteristic length scales and shapefinders are given in the caption of Fig. 7, and they satisfy the inequality $L \geq W \geq T$. 


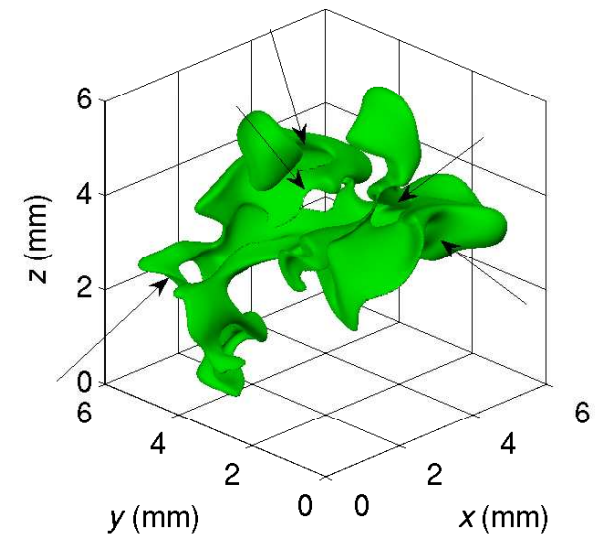

(a)

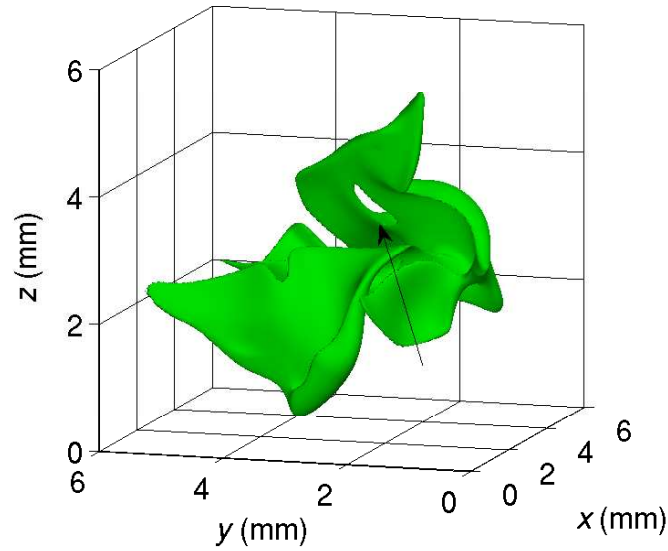

(b)

Figure 7. Two objects chosen arbitrarily are shown above and arrows indicate the locations of holes on them. The values of their Minkowski functionals and shapefinders are as follows. For the extracted object in (a) $V_{0}=8.1 \mathrm{~mm}^{3}, V_{1}=$ $14.5 \mathrm{~mm}^{2}, V_{2}=11.0 \mathrm{~mm}, V_{3}=-8.0(G=5), T=0.28 \mathrm{~mm}, W=0.84 \mathrm{~mm}$, $L=1.4 \mathrm{~mm},(P, F)=(0.50,0.25)$. For the object in (b) $V_{0}=4.6 \mathrm{~mm}^{3}, V_{1}=$ $9.1 \mathrm{~mm}^{2}, V_{2}=6.7 \mathrm{~mm}, V_{3}=0.0 \quad(G=1), T=0.26 \mathrm{~mm}, W=0.87 \mathrm{~mm}$, $L=2.5 \mathrm{~mm},(P, F)=(0.54,0.49)$. 


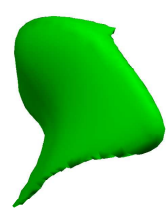

(a) $(0.44,0.15)$.

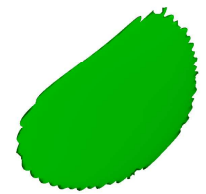

(f) $(0.68,0.24)$.

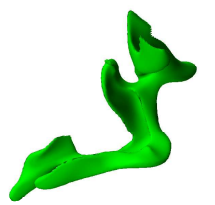

(b) $(0.35,0.69)$.

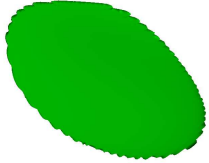

(g) $(0.70,0.18)$.

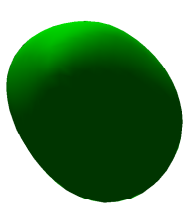

(c) $(0.05,0.05)$.

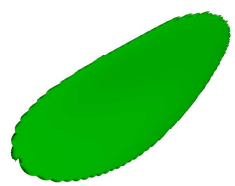

(h) $(0.70,0.19)$.

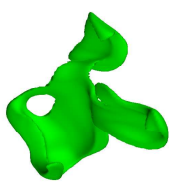

(d) $(0.48,0.42)$.

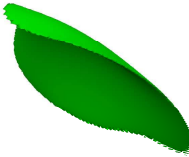

(i) $(0.81,0.19)$.

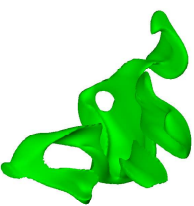

(e) $(0.46,0.62)$.

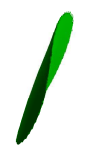

(j) $(0.67,0.23)$.

Figure 8. Samples of reaction zone shapes extracted from the DNS of Case B1 (a to e) and Case $\mathrm{C}$ (f to $\mathrm{j}$ ) at an arbitrary time. The values of their shapefinders, $(P, F)$, are also given above.

The object shown in Fig. 7a presents five holes, indicated by the arrows, and this implies that $V_{3} \ll 0$ as noted in the previous subsection. In this case, Eq. (8) is used to compute $L$. The values of shapefinders, $P$ and $F$, clearly suggests that these objects are pancake-like in the shapefinders map shown in Fig. 5. Thus, the morphology of the complex shaped reaction zones can be identified unambiguously and analysed statistically.

Figure 8 shows arbitrarily chosen typical shapes of reaction zones along with the values of their shapefinders, $(P, F)$, for the MILD and premixed cases considered here. This analysis is conducted using half the number of data sets collected and including the full data sets does not changes the statistics to be discussed in the remainder of this subsection unduly. There are 510, 328, 272 and 42 objects respectively for Cases A1, A2, B1 and C for the statistical analysis of morphological characteristics. Since the reaction rate threshold, $\xi_{1}^{+}$, used is relatively large, even the statistically planar premixed flame has objects enclosed by surfaces not 


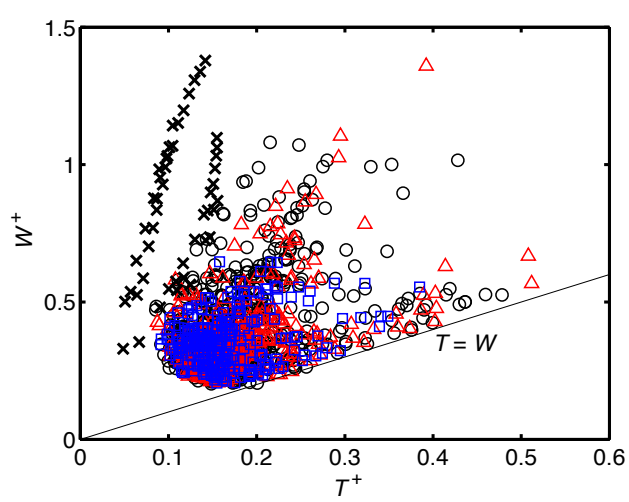

(a)

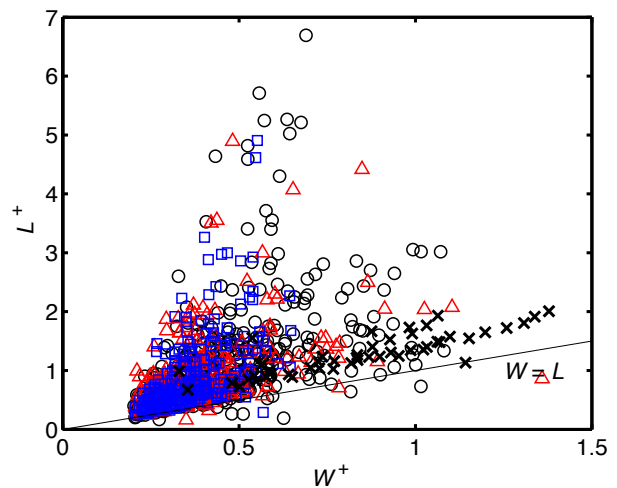

(b)

Figure 9. The three length scales, calculated using Eqs. (5), (6), and (8), for the reaction zones in Case A1 (०), Case A2 $(\triangle)$, Case B1 $(\square)$ and Case $\mathrm{C}(\times)$.

interacting with boundaries. These objects are sheet-like as in Figs. 8f to 8j for the premixed case and their shapes are varied, complex and multiply connected for the MILD combustion cases. It is worth noting that there are spherical reaction zones in the MILD combustion as in Fig. 8c clearly indicated by the low $(P, F)$ values.

Figure 9 shows the three length scales, $L, W$ and $T$, normalised using the respective $\delta_{t h}$ for the MILD and premixed cases. The black solid lines are for $W^{+}=T^{+}$and $L^{+}=W^{+}$and are used to verify whether the inequality $T^{+} \leq$ $W^{+} \leq L^{+}$is met by the computed results in order to have meaningful $P$ and $F$. As one observes in Fig. 9, this inequality is satisfied predominantly and there are some samples violating the condition $L^{+}>W^{+}$. A detailed investigation of these samples identified that this is due to numerical errors as these objects are small and they are excluded from further consideration since they give negative $F$ (see Eq. 10) which is non-physical. The effect of spatial resolution on the calculation 
of the shapefinders is explored in Appendix A. Furthermore, it has been observed that only a very small percentage of the reaction zone structures extracted from the DNS does not obey the inequality. These percentages are 1.8\% in Case A1, 1.2\% in Case A2, 0.37\% in Case B1 and none in Case C. Thus, it becomes clear that the statistics reported here are not influenced by omitting these small structures or numerical errors while calculating $P$ and $F$.

Figure 9 shows that $W^{+}$is much larger than $T^{+}$and it is comparable to $L^{+}$ for Case $\mathrm{C}$, which is the premixed combustion case. This variation suggests that the extracted reaction zone structures are thin sheets as one would expect in premixed combustion. The characteristic thickness for the premixed reaction zones vary from $0.05 \leq T^{+} \leq 0.15$, which is relatively narrow. Their characteristic length and width, however, vary over a wide range as shown in figure $9 \mathrm{~b}$ and the data follow $W=L$ line closely, suggesting a thin sheet. The range for these characteristic scales is wider for the MILD combustion cases as one sees in Fig. 9. The typical ranges are $0.09 \leq T^{+} \leq 0.5,0.2 \leq W^{+} \leq 1.2$ and $0.2 \leq L^{+} \leq 5.5$. These ranges are reduced relatively in Cases A2 and B1 compared to Case A1. As noted in table 2, Cases A2 has a lower turbulence Reynolds number compared to Case A1 despite the same dilution level and thus the range of these scales are reduced somewhat. However, for the most diluted Case B1 having the same $\operatorname{Re}_{l 0}$ as Case A1, most of the data are concentrated in a relatively narrower range. This is because of mixture non-uniformity in Case B1 resulting in convolutions and interactions of reaction zones as shown in Fig. 4. The characteristics of sheet-like structures seen in the premixed case is not observed for the MILD combustion cases.

The characteristic scales shown in Fig. 9 are used to calculate the shapefinders 


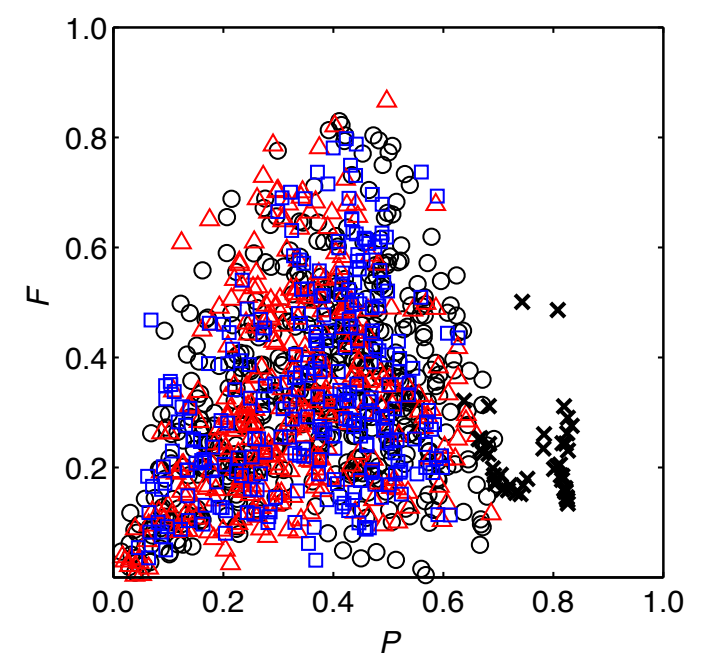

Figure 10. Shapefinder, $P$ and $F$, for Case A1 (o), Case A2 ( $\triangle)$, Case B1 ( $\square)$, and Case $\mathrm{C}(\times)$.

$P$ and $F$ using Eqs. (9) and (10), which are shown in Fig. 10. The results for the premixed case reside in a narrow region mostly confined to the $(P, F)$ regions for sheet-like structures, but the MILD reaction zones have a broader distribution with about $0.01 \leq P \leq 0.7$ and $0.01 \leq F \leq 0.8$. A comparison of these results to Fig. 5 suggests that the MILD reaction zone shapes range from blobs (small $P$ and $F$ ) to pancakes (intermediate $P$ and $F$ ). There are some short ribbons also and the pocket burnouts would yield a blob-like shape represented by the points close to $(0,0)$ in Fig. 10. Representative pictures of these structures as extracted from the DNS data are shown in Fig. 8.

The marginal PDF of $P$ and $F$ constructed using 20 bins for the MILD cases and 12 bins for the premixed case shown in Fig. 11 suggests that $P$ and $F$ are distributed from about 0.01 to 0.8 for the MILD cases as discussed above. However for the premixed case, their ranges are $0.6 \leq P \leq 0.9$ and $0.1 \leq F \leq 0.4$, 


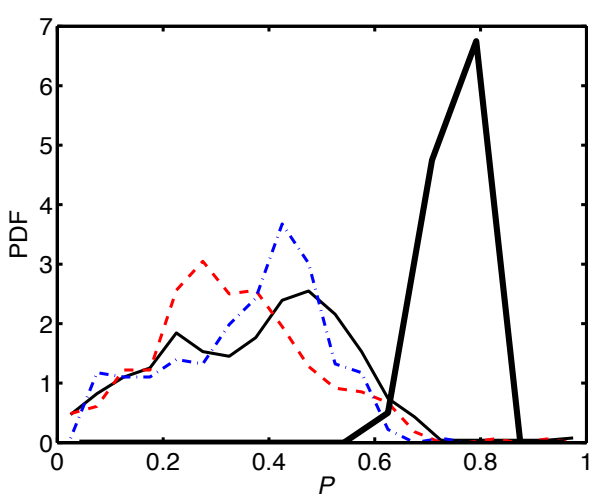

(a)

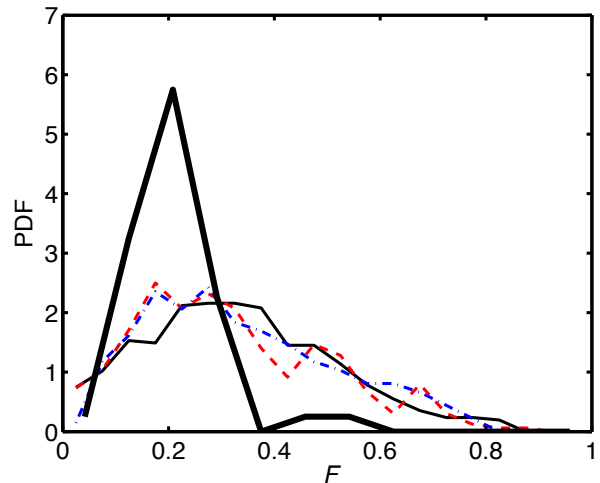

(b)

Figure 11. PDF of (a) planarity and (b) filamentarity for Case A1 (thin-solid), Case A2 (dashed), Case B1 (dash-dotted), and Case C (thick solid).

with pronounced peaks in the PDFs as seen in Fig. 11. This figure suggests that the most probable value for $(P, F)$ is $(0.79,0.2)$ for the premixed case. The most probable value of $P$ varies between 0.3 and 0.5 , and $F$ is about 0.2 for the MILD cases. These values suggest that the most probable shape for the MILD reaction zone is pancake-like. Such shapes can result from autoigniting regions which do not necessarily result in thin flame fronts. The average and most probable values of volume $\mathcal{V}$ and the three characteristic length scales of these structures used in the above analyses are given in Table 3. These values are normalised using the respective $\delta_{t h}$ given in the same table. The mean and most probable $T^{+}$of the reaction zones show a very weak sensitivity to Da, meaning the dimensional thickness increases with dilution level since $\delta_{t h}$ increases. The values of $W_{\text {mean }}^{+}$ and $L_{\text {mean }}^{+}$also show this insensitivity in the MILD cases. The value of $\mathcal{V}_{\text {mean }}^{+}$for Case A1 is about twice as large as for Case A2, while the values of $\mathcal{V}_{\text {prob }}^{+}$are identical for these two cases. This implies that a substantial number of local reaction 
Table 3. Average and most probable values of $\mathcal{V}$ and the three characteristic length scales (Eqs 5-7 and 8).

\begin{tabular}{c||ccccccccccc}
\hline & $\delta_{t h}(\mathrm{~mm})$ & $\mathrm{Da}$ & $\mathrm{Ka}$ & $\mathcal{V}_{\text {mean }}^{+}$ & $T_{\text {mean }}^{+}$ & $W_{\text {mean }}^{+}$ & $L_{\text {mean }}^{+}$ & $\mathcal{V}_{\text {prob }}^{+}$ & $T_{\text {prob }}^{+}$ & $W_{\text {prob }}^{+}$ & $L_{\text {prob }}^{+}$ \\
\hline Case A1 & 1.00 & 1.72 & 4.78 & 0.93 & 0.19 & 0.44 & 1.00 & 0.07 & 0.15 & 0.26 & 0.46 \\
Case A2 & 1.00 & 3.25 & 2.11 & 0.42 & 0.19 & 0.38 & 0.87 & 0.07 & 0.18 & 0.29 & 0.45 \\
Case B1 & 1.29 & 0.69 & 11.9 & 0.30 & 0.17 & 0.36 & 0.85 & 0.12 & 0.16 & 0.36 & 0.63 \\
Case C & 0.37 & 5.64 & 0.92 & 0.56 & 0.11 & 0.84 & 1.27 & 0.22 & 0.15 & 0.83 & 1.23 \\
\hline
\end{tabular}

zones have larger volumes in Case A1 due to frequent reaction zone interactions resulting from higher turbulence level although these two cases have equal number (in a probability sense) patchy reaction zones having $\mathcal{V}^{+} \sim 0.07$.

Generally $L_{\text {mean }}^{+}$is larger than $W_{\text {mean }}^{+}$and the ratio $L_{\text {mean }}^{+} / W_{\text {mean }}^{+}$is about 2.3 for the MILD cases and 1.5 for the premixed case. The ratio $W_{\text {mean }}^{+} / T_{\text {mean }}^{+}$is also about 2.1 for the MILD cases, but it is 7.6 for the premixed case. These values clearly agree with the view that the MILD reaction zones are not thin sheets even in an average sense. Although the local reaction zone volume $\mathcal{V}^{+}$would change with the threshold value $\xi_{1}^{+}$, the computed value of $\mathcal{V}^{+}$varies due to dilution level under the same turbulence condition (compare Cases A1 and B1). This suggests that the widely used Eddy Dissipation Concept (EDC) model could be improved further for MILD combustion if the volume of local reacting regions is determined not only by the size of turbulence energy dissipation regions, but also by considering the effect of dilution on characteristic scales such as $\mathcal{V}^{+}$.

\subsection{Autoignition and flame propagation characteristics}

The analysis in the previous subsection showed that MILD reaction zones have statistically pancake-like shapes. Since propagating flame is usually associated with thin sheet-like structures, these pancake-like shapes are suggestive of 
frequent events of autoignition of interacting flames in MILD combustion. Autoignition is sensitive to the local temperature and mixture composition which are influenced by turbulent mixing and molecular diffusion [3, 51, 17]. Especially, turbulent mixing directly affects the autoignition delay time [51]. In the present configuration, inlet mixture is imperfectly mixed containing reactants and products of EGR mixtures and turbulence resulting in spatio-temporal variation of local mass fractions of chemically active radicals and intermediates. This results in spatial variation of autoignition delay time. However, the local turbulence conditions influence the local residence time for the mixture which may result in insufficient time for autoignition but may create favourable conditions for propagating flames or vice-versa. The influences of global mixture composition on autoignition have been investigated for MILD combustion in the past [52]. However, competing effects of local turbulence and mixture composition on autoignition or propagating flame characteristics have not been studied although these characteristics are likely to determine the type of modelling used for MILD combustion. The predominant characteristics of local combustion in MILD mixtures is studied using a flux-balance analysis in the next subsection.

\subsubsection{Balance of convection, diffusion and chemical reaction}

Turbulent combustion involves a balance of convection, diffusion and chemical reaction processes. The transport equation for normalised temperature, $c_{T}$, is written as:

$$
\frac{\partial \rho c_{T}}{\partial t} \underbrace{+\frac{\partial \rho u_{i} c_{T}}{\partial x_{i}}}_{\mathscr{C}: \text { convection }}=\underbrace{\frac{\partial}{\partial x_{j}}\left(\rho D_{c_{T}} \frac{\partial c_{T}}{\partial x_{j}}\right)}_{\mathscr{D}: \text { diffusion }}+\underbrace{\omega_{c_{T}}}_{\mathscr{R}: \text { reaction }},
$$


where $u_{i}$ is the fluid velocity in direction $x_{i}$ and $D_{c_{T}}$ is the molecular diffusivity of $c_{T}$. The balance among these terms, $\mathscr{C}, \mathscr{D}$ and $\mathscr{R}$, may be assessed by studying $\mathscr{B}$ defined as:

$$
\mathscr{B} \equiv|\mathscr{C}-\mathscr{D}|-|\mathscr{R}|,
$$

which makes Eq. (11) read as $\partial \rho c_{T} / \partial t+\mathscr{B}=0$. For zero-dimensional homogeneous transient combustion in a fixed-mass constant pressure reactor, Eq. (11) is dominated by the chemical source $\mathscr{R}$ and unsteady terms, giving $\mathscr{B}=-|\mathscr{R}|$. This situation is akin to combustion in a Perfectly Stirred Reactor (PSR) with a volume $V$ and a mass flow rate of $\dot{m}_{i n}$, having a governing equation for $c_{T}$ given by

$$
\frac{d c_{T}}{d t}=-\frac{c_{T}}{\tau_{R}}+\frac{\omega_{c_{T}}}{\rho}
$$

where $\tau_{R}$ is the residence time of a reactor defined as $\tau_{R}=\rho V / \dot{m}_{i n}$. Thus, for a model configuration such as homogeneous reactor or PSR, regions with $\mathscr{B} \ll 0$ signify autoigniting events, where the transport equation of $c_{T}$ is dominated by the unsteady and chemical source terms. Although it may be inappropriate to consider the entire MILD combustion as a homogeneous reactor, the elevated temperature of reactant mixture and distributed reaction zones discussed in section 3.1 may generate local reaction zones having $\mathscr{B} \ll 0$. In the present MILD cases, not only autoigniting mixtures but also regions with flame interactions have small $\mathscr{C}$ and $\mathscr{D}$ yielding negative $\mathscr{B}[12]$. Thus, regions with $\mathscr{B}<0$ are called reaction dominated regions in this study in order to include autoignition and interacting flames. Clearly, such regions cannot be modelled using a standard flamelet approach involving scalar gradient related quantities, since the direct relation between the scalar gradient and reaction rate no longer holds for these and also there is no 
convective-diffusive-reactive balance.

Another canonical model is a steady one-dimensional premixed flame with inlet mixture velocity of $S_{L}$. In this case, a balance among the convective, diffusive and reactive terms gives $\mathscr{B}=0$ and the reactive contribution scales as $\rho_{r} S_{L} / \delta_{t h}$. In turbulent premixed flames, however, there are large velocity fluctuations leading to local displacement of the flame and thus large $\mathscr{C}$ and $\mathscr{R}$ are observed near flame fronts yielding $\mathscr{B}>0$. This type of reaction zone might be similar to those observed under Homogeneous Charge Diffusion Ignition (HCDI) conditions [53].

Based on these insights, the reaction and propagating-flame dominated regions are identified using $\mathscr{B}<0$ and $\mathscr{B}>0$ respectively. Although, ignition front and flame fronts may be identified by computing displacement speed [54] or by using Chemical Explosive Mode Analysis (CEMA) [55], the present analysis employs above criteria to connect the identification more directly with the canonical ignition and propagation phenomena for future modelling purposes.

Figure 12a shows typical contours of $\left|\mathscr{C}^{+}-\mathscr{D}^{+}\right|=1.5$ (dashed lines) and $\left|\mathscr{R}^{+}\right|=1.5$ (solid lines) in a $y$ - $z$ plane at $x^{+} \approx 0$ (one grid point inside the computational domain) for Case B1. The contours of $\left|\mathscr{C}^{+}-\mathscr{D}^{+}\right|=1.5$ are wrinkled and do not seem to exactly follow the contours of $\left|\mathscr{R}^{+}\right|=1.5$ although a steady laminar flame solution is used to generate the initial and inlet fields as described in section 2.2. This is due to the role of turbulent mixing and molecular diffusion that would have occurred in the final preprocessing step discussed in section 2.2. However, these contours seem to cluster at similar locations as in Fig. 12a.

The comparison of these two contours becomes more interesting if one considers a $y-z$ plane at a downstream position. These contours are shown in Fig. 12b 


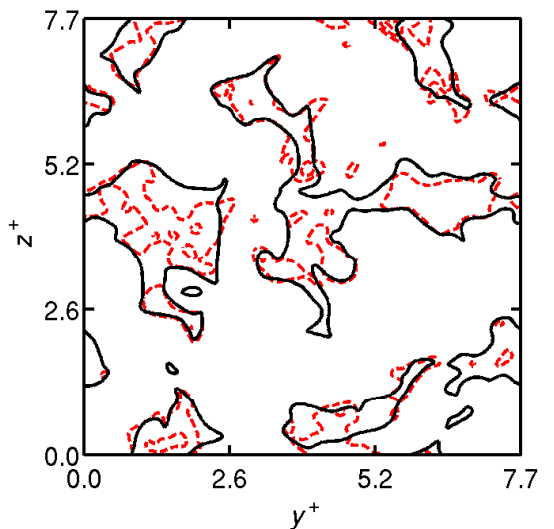

(a)

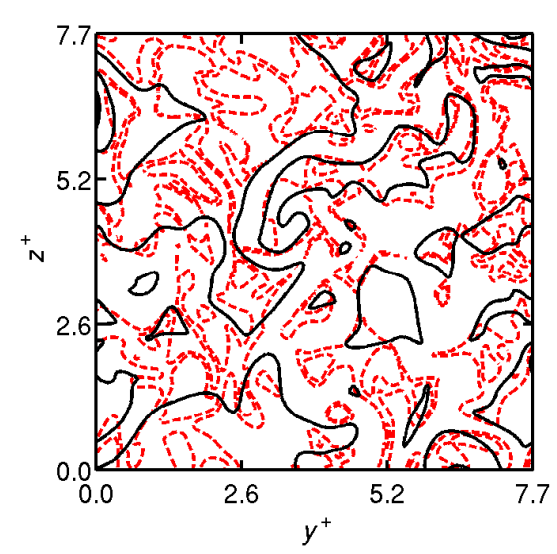

(b)

Figure 12. Typical contours of $\left|\mathscr{C}^{+}-\mathscr{D}^{+}\right|=1.5$ (dashed line) and $\left|\mathscr{R}^{+}\right|=1.5$ (solid) in a $y-z$ plane. These planes are extracted from an instantaneous 3D field obtained at $t=1.5 \tau_{D}$, and the locations are (a) $x^{+}=0$ and (b) 2.6.

for the $y-z$ plane located at $x=L_{x} / 3$. The contours of $\left|\mathscr{R}^{+}\right|=1.5$ correspond to $\left|\mathscr{C}^{+}-\mathscr{D}^{+}\right|=1.5$ contours in some regions signifying propagating flames locally because of the convective-diffusive-reactive balance. One also observes regions of predominant convection-diffusion and reaction alone without convectiondiffusion. This reactive region signifies the presence of autoignition or interacting flames. The spatial difference among these contours also suggests a strong spatial variation for the unsteady term in Eq. (11).

The local dominance of flame propagation or reaction is studied by investigating the spatial variation of $\mathscr{B}^{+}$. The typical spatial variation is shown in Fig. 13 as $\mathscr{B}^{+}=1.5$ and -1.5 contours along with $\omega_{c_{T}}^{+}$. This result is shown for Case B1 and it is similar for the other two MILD combustion cases. The reaction dominated regions having $\mathscr{B}^{+}<0$ as noted earlier in this subsection are marked using the black contour lines. One of the reaction dominated regions hav- 
ing $\mathscr{B}^{+}<0$ is marked using a black box with solid lines, which is enlarged for clarity (see Fig. 13, bottom right picture). As one gathers from this enlarged inset, $\mathscr{B}^{+}<-1.5$ when $\omega_{c_{T}}^{+}$is large. The propagating-flame dominated regions, $\mathscr{B}^{+}>0$, are marked using the white contour lines and one such region is marked by a white box, which is also enlarged for clear exposition of $\mathscr{B}^{+}>1.5$ with intense reactions signifying the presence of propagating-flames, see Fig. 13, top right picture.

There are many reaction zones having $\mathscr{B}^{+}>0$ for some parts of them and $\mathscr{B}^{+}<0$ for the rest. Four such regions are marked using black boxes with dashed lines. Also, there are regions with entangled reaction-dominated and propagating-flame attributes. The reaction dominated events are closely followed by flame propagation. The central region marked using a box with dash-dotted line in Fig. 13 is a good example for such a region with both reaction-dominated and propagating-flame phenomena occurring close by. This region is also enlarged in the middle right picture of Fig. 13. The variation of $\mathscr{B}^{+}$depicted in Fig. 13 shows that both reaction and propagating-flame dominated regions coexist in MILD combustion.

The statistical nature of this coexistence can be investigated using the PDF of $\mathscr{B}^{+}$and if this coexistence is statistically dominant then this PDF is expected to be uniform. This PDF, typical for the MILD combustion cases studied here, is shown in Fig. 14 for various streamwise positions from the Case B1. This PDF for the premixed undiluted case is also shown in Fig. 14 for $x^{+}=10.2$ for comparison. The variation of the most probable (circles) and averaged (triangles) $\mathscr{B}^{+}$with $x^{+}$ is shown in the inset. The behaviour of $P\left(\mathscr{B}^{+}\right)$in the premixed undiluted case shows a peak close to zero signifying the balance among convection, diffusion 


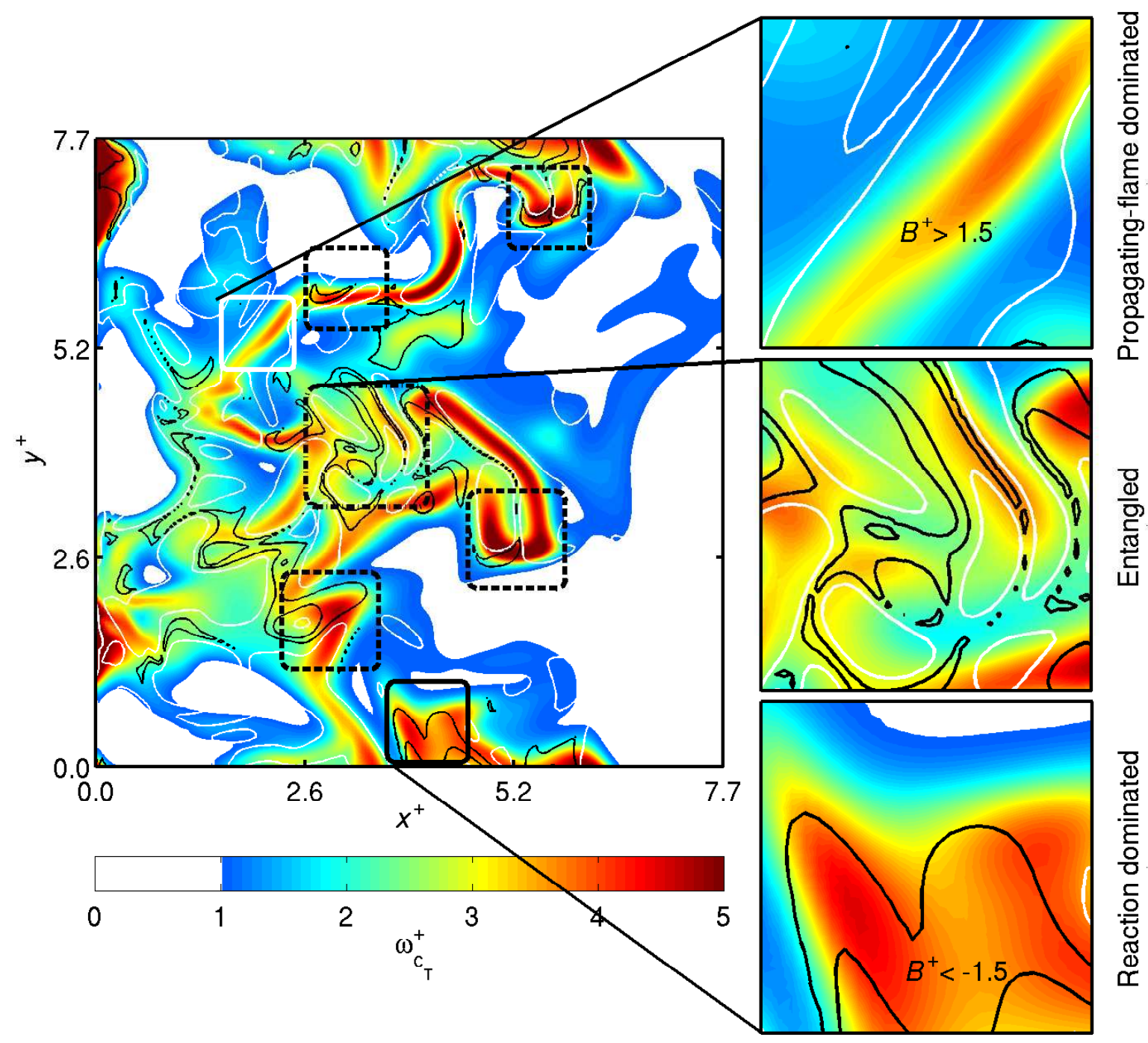

Figure 13. Typical contours of $\omega_{c_{T}}^{+}$(colour map) are shown along with propagating-flame dominated $\left(\mathscr{B}^{+}=1.5\right.$, white contour lines) and reaction dominated $\left(\mathscr{B}^{+}=-1.5\right.$, black contour lines) regions for Case B1 in the same $x-y$ plane as in Fig. 4d. A typical reaction dominated and propagating-flame dominated regions are marked respectively using a black box and a white box with solid lines, which are enlarged at bottom right and top right respectively. Several continuous reaction zones having both reaction and propagating-flame phenomena are marked using black boxes with dashed lines. The box with dash-dotted lines in the centre denotes a region where the two phenomena are closely entangled, which is also enlarged at the middberight. 
and reaction in an average sense. The PDF is broader for the MILD case and it depends on the streamwise position, $x^{+}$. The peak of $P\left(\mathscr{B}^{+}\right)$is close to $\mathscr{B}^{+} \approx 0$ for positions close to the inlet boundary. This peak shifts to negative $\mathscr{B}^{+}$and then moves towards $\mathscr{B}^{+}=0$ as the sampling position moves downstream. These changes are gradual as shown in the inset in Fig. 14. The most probable $\mathscr{B}^{+}$, however, stays negative as in the inset but the averaged $\mathscr{B}^{+}$is positive for various streamwise positions considered. Thus, the MILD combustion is dominated by strong unsteady propagating-flame behaviour in an average sense although there are many reaction dominated regions locally. The long positive tail of $P\left(\mathscr{B}^{+}\right)$resulting from convection-diffusion dominated regions yields $\overline{\mathscr{B}^{+}}>0$. Similar behaviour of this PDF is also observed if the progress variable is defined using mass fraction of $\mathrm{CH}_{4}, \mathrm{H}_{2} \mathrm{O}$ or $\mathrm{CO}_{2}$. The existence of both reaction and propagatingflame dominated regions along with a broad distribution of $P\left(\mathscr{B}^{+}\right)$is also seen irrespective of the progress variable definition. This signifies the coexistence of reaction and propagating-flame dominated phenomena along with the importance of strong local unsteady effects in the MILD combustion.

It is well known that the gradient of $c_{T}$ plays an important role in turbulent premixed combustion $[56,57,58]$. Thus, the scalar gradient magnitude is expected to be large in propagating-flame dominated regions having $\mathscr{B}^{+} \geq 0$. The contours of scalar gradient normalised using $\delta_{t h}$ are shown in Fig. 15 for Case B1 along with the contours of $\mathscr{B}^{+}=1.5$ and -1.5 . The scalar gradient field is only shown for the regions with $\omega_{c_{T}} \geq 1.0$ so that a direct comparison with Fig. 13 can be made. The regions of high reaction rate are not necessarily associated with regions of large scalar gradient in MILD combustion due to interactions of reaction zones [12] unlike in premixed combustion [57,59]. This can be seen by compar- 


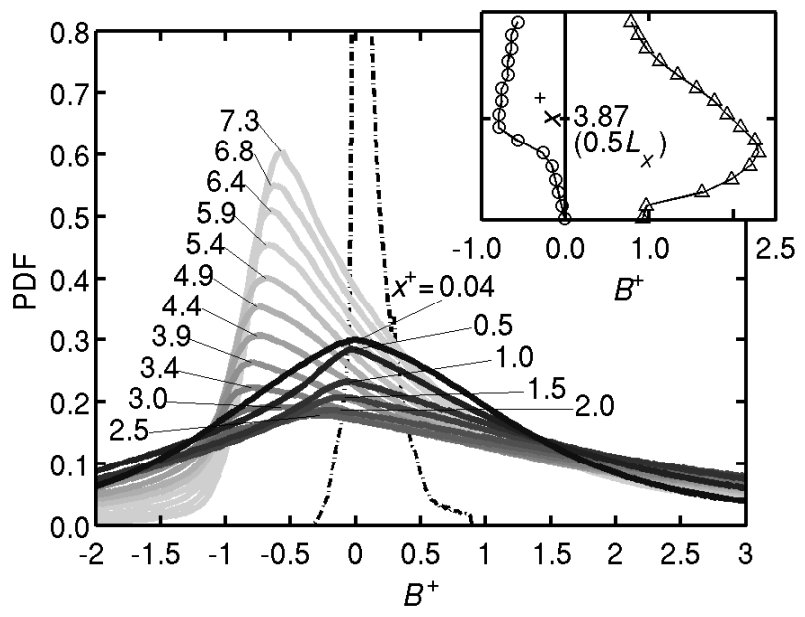

Figure 14. PDF of $\mathscr{B}^{+}$at various $x^{+}$positions in Case B1. The PDF is also shown for the premixed case at $x^{+}=10.2$ (dash-dotted line). The inset shows the variation of the most probable (०) and averaged $(\triangle)$ values of $\mathscr{B}^{+}$along $x^{+}$.

ing Figs. 13 and 15. Also, the regions of large scalar gradients are mostly enclosed by white lines for $\mathscr{B}^{+}=1.5$ denoting propagating-flame dominated regions. The substantial part of the reaction dominated regions denoted by black lines in Fig. 15 have relatively small scalar gradient. These results further reinforce the observation on the distinctive scalar gradient characteristics of the MILD combustion in a previous study [24].

In premixed combustion, intense heat release rate is associated with large scalar dissipation rate, which is directly related to scalar gradient magnitude [57, $58,59]$. Thus, regions with large scalar gradient are conducive to the establishment of propagating flames which propagate until they collide with other locally propagating flames [12]. The propagating flames may not established quickly in regions with small scalar gradient which may be conducive to establish reaction-dominated events and thus these events are prevalent in these loca- 
tions for MILD mixtures. Similar observations for reaction dominated events have been reported in earlier studies of non-premixed and partially-premixed combustion $[60,61,62,17]$.

Scalar gradient characteristics in reaction and propagating-flame dominated regions can be studied statistically using the PDFs of $\omega_{c_{T}}^{+}$and $\left|\nabla c_{T}\right|^{+}$conditioned on $\mathscr{B}^{+}$. Here, two conditions $\mathscr{B}^{+} \leq-1.5$ for reaction dominated and $\mathscr{B}^{+} \geq 1.5$ for propagating-flame dominated regions are used. These PDFs are shown in Fig. 16 for various $x^{+}$positions for Case B1. These PDFs are constructed using all the data sampled for this case.

The PDF of $\omega_{c_{T}}^{+}$conditioned for two conditions of $\mathscr{B}^{+}$is depicted in Fig. 16a. It shows that the range of $\omega_{c_{T}}^{+}$is broader for reaction dominated regions compared to the propagating-flame dominated regions and also there is a clear separation in the range of $\omega_{c_{T}}^{+}$. The PDF peaks around $\omega_{c_{T}}^{+}=1.0$ for the propagating-flame dominated regions since $\omega_{c_{T}}^{+}$scales as $\rho_{r} S_{L} / \delta_{t h}$ for the premixed flamelets. There is a small decrease of the value of $\omega_{c_{T}}^{+}$as one moves downstream inside the computational domain. The PDFs are relatively broader and the average value of $\omega_{c_{T}}^{+}$ is significantly larger for the reaction dominated regions. The peak of the PDF decreases as $x^{+}$increases, but the range of $\omega_{c_{T}}^{+}$having non-zero probability increases suggesting a more uniform distribution of reaction dominated regions in the downstream part of the MILD combustion domain.

The conditioned PDFs for $\left|\nabla c_{T}\right|^{+}$are shown in Fig. 16b. Although the range of $\left|\nabla c_{T}\right|^{+}$is similar for reaction and propagating-flame dominated regions there seem to be some preferred scalar gradient values for these phenomena. The most probable scalar gradient in reaction dominated regions is generally smaller than that in propagating-flame dominated regions. This offers further support to the 


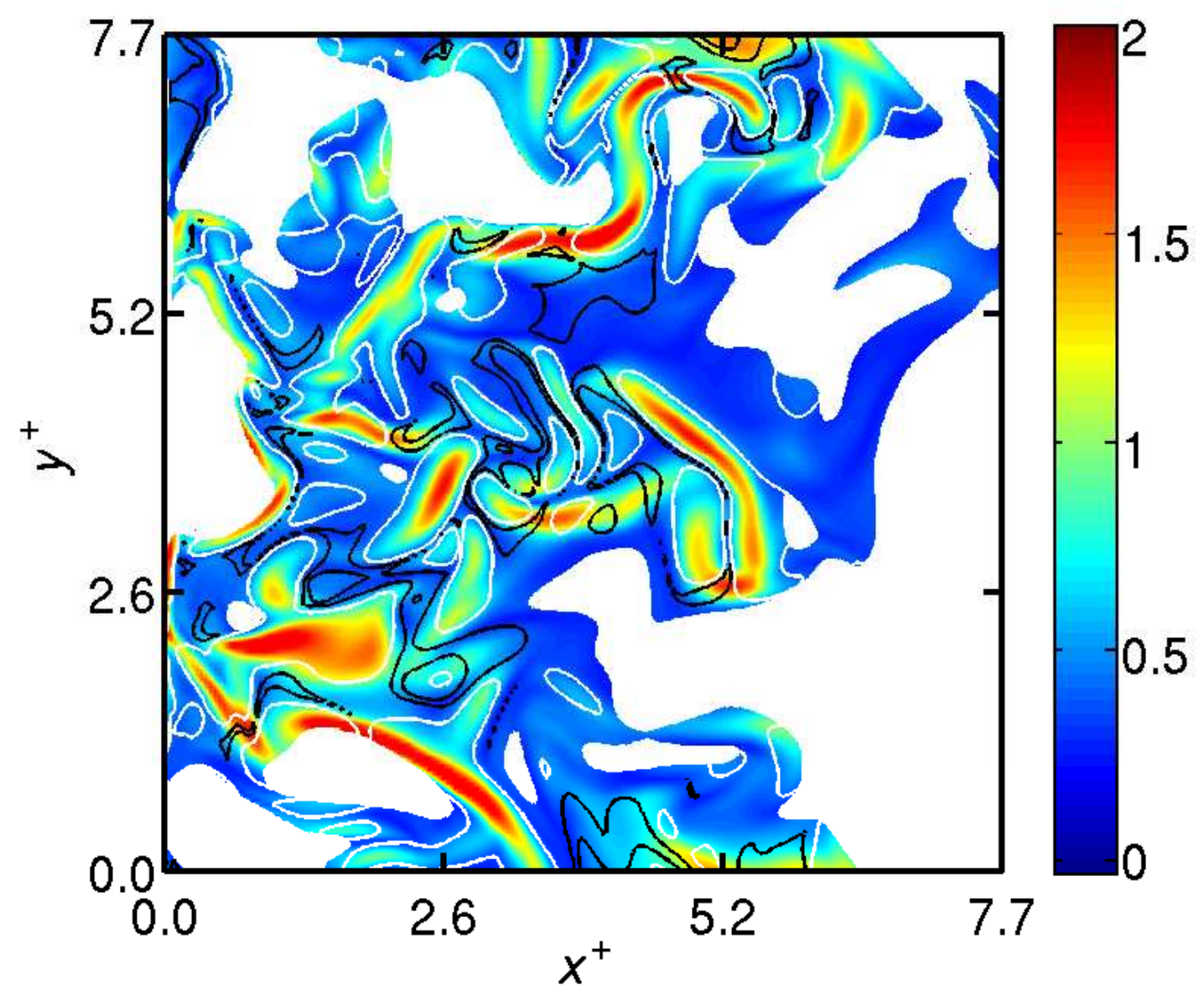

Figure 15. Typical contours of $\left|\nabla c_{T}\right|^{+}$(colour map) are shown along with propagating-flame dominated $\left(\mathscr{B}^{+}=1.5\right.$, white contour lines) and reaction dominated $\left(\mathscr{B}^{+}=-1.5\right.$, black contour lines) regions for Case B1 in the mid $x$-y plane at $t=1.5 \tau_{D}$. Regions with $\omega_{c_{T}}^{+} \leq 1.0$ are masked using white colour in the same way as in Fig. 13. 


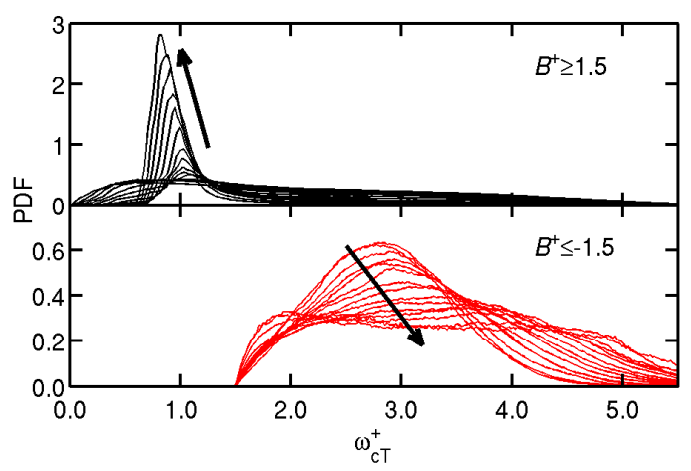

(a)

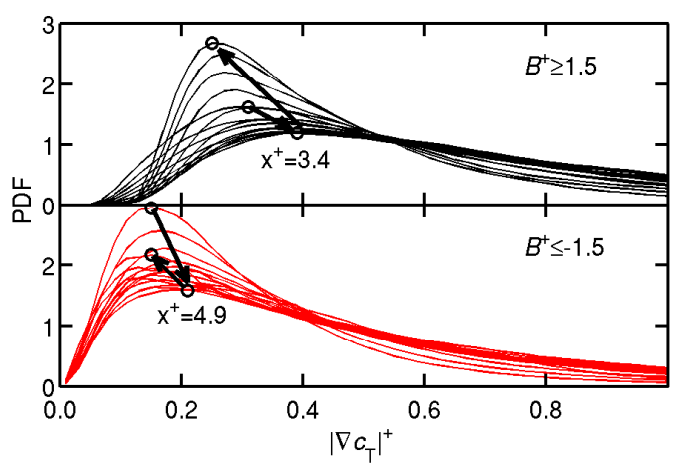

(b)

Figure 16. PDFs of (a) $\omega_{c_{T}}^{+}$and (b) $\left|\nabla c_{T}\right|^{+}$in propagating-flame dominated regions, $\mathscr{B}^{+} \geq 1.5$, and reaction dominated regions, $\mathscr{B}^{+} \leq-1.5$, for various $x^{+}$ positions given in Fig. 14. Arrows indicate the general shift of the PDF peaks with $x^{+}$. The results are typical for MILD cases.

physical picture gleaned from the DNS data discussed above for the physics of MILD combustion. Therefore, in the present MILD combustion conditions, both reaction and propagating-flame dominated regions coexist. Despite this, the local scalar gradient resulting from the mixture distribution, turbulence and molecular diffusion decides whether reaction dominated or propagating-flame dominated activities are favoured locally.

\section{Conclusions}

Three-dimensional DNS results of turbulent MILD and conventional premixed combustion have been studied to understand the fundamental physics of reaction zones and their morphology in MILD combustion. The results of the premixed case is used for comparative analyses. The DNS configuration includes inflow, outflow and periodic boundaries and the inflow mixture contains pockets of reac- 
tants and products to represent mixtures for MILD combustion.

Generally, MILD combustion is called flameless combustion implying that it does not involve intense reaction zones. This study using DNS data clearly demonstrates that there are regions with strong chemical activities and these regions are distributed over a good portion of the computational domain unlike in the premixed flame case where the reaction zones are confined to a small portion of the domain. Also, interactions of reaction zones are observed for the conditions of MILD combustion investigated in this study, and the frequency and extent of this interaction seem to increase with dilution. These interactions yield an appearance of distributed turbulent combustion under MILD conditions and thus this combustion does seem flameless.

The morphology of the reaction zones in the MILD and premixed combustion is also investigated using Minkowski functionals. There are four functionals for a three dimensional object, and three characteristic length scales for the object are deduced using these four functionals. A shapefinder map involving two parameters, planarity and filamentarity, is obtained using the three characteristic length scales. These shapefinders are used for a convenient and unambiguous morphological classification of the reaction zone structures observed in the DNS as detailed in section 3.2. These structures are extracted using the reaction rate value, $\xi_{1}$, corresponding to the maximum of $\mathcal{Z}(\xi)=\left\langle\Delta S N_{c} \omega_{c_{T}} \mid \omega_{c_{T}}=\xi\right\rangle$, which is the conditionally averaged value of the reaction rate weighted by the scalar dissipation rate and surface area. This avoids subjectivity that may arise if one uses a simple threshold value. From this analysis, predominantly sheet-like structures are observed for the premixed combustion case as one would expect. However, the MILD reaction zones show varied shapes, blobs, pancakes, small ribbons, 
etc., made of simply and multiply connected objects. The statistical analysis of the shapefinders for the MILD reaction zones suggests that a pancake-like structure is the most probable shape, which results from events such as autoignition, propagating-flames of mixtures containing reactants and products with radicals and intermediates, and interactions of these flames. These phenomena are also entangled in space and time and separating them for modelling purposes may not be easy. However, as noted in Sec. 3.2.2, conventional turbulent combustion models, such as the EDC model, could be improved by considering changes in local reaction zone volumes due to these phenomena for MILD combustion.

The various fluxes involved in the progress variable transport equation are also analysed for their spatial variations at a given instant and also statistically to shed more light on the above observations. This analysis supports the above insight, suggesting that there are regions dominated by reaction and propagatingflame phenomena along with their coexistence for the conditions investigated in this study. Although, these regions are entangled closely, the local behaviour of scalar gradient affects whether reaction or propagating-flame dominated activities are favoured locally. These activities produce reaction zones of varied shapes and sizes in MILD combustion and these are analysed using Minkowski functionals as noted above. These analyses show that there are reaction zones with holes resulting from contortion of reaction zones and their interactions because of turbulence. Local extinction cannot be the cause for these holes to form because local extinction does not occur for the conditions considered in this study. These interacting reaction zones with single or multiple holes offer new challenges for turbulent combustion modelling. The turbulence-chemistry interaction may have some dependence on the chemical kinetic mechanism and thus the use of a more detailed 
kinetic mechanism for the DNS is of some interest for further investigation. However, the broader perspectives and implications of the observations made in this study is less likely to change.

The relative role of autoignition and flame-propagation may change with a decrease in $T_{r}$ and one needs further direct simulations to assess this conclusively. It is also imperative that the combustion sub-modelling for turbulent combustion under MILD conditions must be able to cater for both autoignition and flamepropagation since these processes are likely to be interweaved in space and time.

\section{Acknowledgements}

YM acknowledges the financial support of Nippon Keidanren and Cambridge Overseas Trust. EPSRC support is acknowledged by NS. This work made use of the facilities of HECToR, the UK's national high-performance computing service, which is provided by UoE HPCx Ltd at the University of Edinburgh, Cray Inc and NAG Ltd, and funded by the Office of Science and Technology through EPSRCs High End Computing Programme. 


\section{Appendix A.}

Three simple shapes, a sphere with radius $r_{s}$, a cylinder with radius $r_{c}$, and an elliptic cylinder with $r_{e 1}$ and $r_{e 2}$ respectively as minor and major radii are tested to determine the spatial resolution required to obtain accurate shapefinders. Figure A.17 shows the variations of $P$ and $F$ for the three test cases as a function of $\Delta \kappa_{m}$, where $\Delta$ is numerical resolution (mesh spacing) and $\kappa_{m}$ is the maximum curvature calculated as $1 / r_{s}$ for the sphere, $1 / r_{c}$ for the cylinder, and $1 / r_{e 1}$ for the elliptic cylinder. For large values of $\Delta \kappa_{m}$, the fluctuations in $P$ and $F$ are large due to the lack of spatial resolution and $\Delta \kappa_{m} \leq 0.10$ yields $P$ and $F$ within $\pm 3.5 \%$ of their actual (theoretical) values. Therefore in this study, any objects having $\Delta \kappa_{m}>0.10$ are excluded from the calculation.

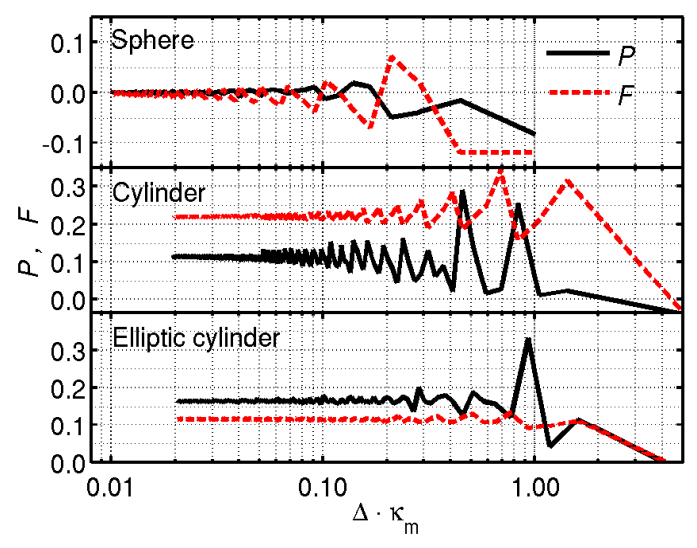

Figure A.17. $P$ and $F$ variations for test cases as a function of numerical resolution. 


\section{References}

[1] J. A. Wünning, J. G. Wünning, Prog. Energy Combust. Sci. 23 (1997) 81-94.

[2] M. Katsuki, T. Hasegawa, Proc. Combust. Inst. (1998) 3135-3146.

[3] A. Cavaliere, M. de Joannon, Prog. Energy Combust. Sci. 30 (2004) 329366.

[4] T. Plessing, N. Peters, J. G. Wünning, Proc. Combust. Inst. (1998) 31973204

[5] P. R. Medwell, P. A. M. Kalt, B. B. Dally, Combust. Flame 148 (2007) 4861.

[6] M. de Joannon, A. Saponaro, A. Cavaliere, Proc. Combust. Inst. 28 (2000) $1639-1646$.

[7] I. B. Özdemir, N. Peters, Exp. Fluids 30 (2001) 683-695.

[8] B. B. Dally, E. Riesmeier, N. Peters, Combust. Flame 137 (2004) 418-431.

[9] N. Krishnamurthy, P. J. Paul, W. Blasiak, Proc. Combust. Inst. 32 (2009) 3139-3146.

[10] S. Hayashi, Y. Mizobuchi, in: N. Swaminathan, K. N. C. Bray (Eds.), Turbulent premixed flames, Cambridge University Press, Cambridge, UK, 2011, pp. 365-378.

[11] C. Duwig, B. Li, M. Aldén, Combust. Flame 159 (2012) 306-316.

[12] Y. Minamoto, T. D. Dunstan, N. Swaminathan, R. S. Cant, Proc. Combust. Inst. 34 (2013) 3231-3238. 
[13] P. R. Medwell, Laser diagnostics in MILD combustion, Ph.D. thesis, The University of Adelaide, Adelaide, Australia, 2007.

[14] P. R. Medwell, P. A. M. Kalt, B. B. Dally, Combust. Flame 152 (2008) 100113.

[15] J. A. van Oijen, Proc. Combust. Inst. 34 (2013) 1163-1171.

[16] E. Mastorakos, A. M. K. P. Taylor, J. H. Whitelaw, Combust. Flame 102 (1995) 101-114.

[17] C. M. Arndt, J. D. Gounder, W. Meier, M. Aigner, Applies Physics B 108 (2012) 407-417.

[18] E. Mastorakos, Prog. Energy Combust. Sci. 35 (2009) 57-97.

[19] R. S. Cant, in: Technical Report CUED/A-THERMO/TR67, Cambridge University Engineering Department, 2012.

[20] T. D. Dunstan, N. Swaminathan, K. N. C. Bray, R. S. Cant, Flow Turbulence Combust. 87 (2011) 237-259.

[21] T. D. Dunstan, N. Swaminathan, K. N. C. Bray, J. Fluid Mech. 709 (2012) $191-222$.

[22] M. D. Smooke, V. Giovangigli, in: M. D. Smooke (Ed.), Reduced kinetic mechanisms and asymptotic approximations for methane-air flames., volume 384, Springer Verlag, New York, 1991, pp. 1-28.

[23] T. Poinsot, S. Lele, J. Comput. Phys. 101 (1992) 104-129. 
[24] Y. Minamoto, N. Swaminathan, Combust. Flame (2013) 10.1016/j.combustflame.2013.10.005.

[25] G. K. Batchelor, A. A. Townsend, Proc. Roy. Soc. Lond. A 194 (1948) 527543.

[26] V. Eswaran, S. B. Pope, Phys. Fluids 31 (1987) 506-520.

[27] R. W. Bilger, S. H. Stårner, R. J. Kee, Combust. Flame 80 (1990) 135-149.

[28] Y. Suzukawa, S. Sugiyama, Y. Hino, M. Ishioka, I. Mori, Energy Convers. Mgmt 38 (1997) 1061-1071.

[29] E. Oldenhof, M. J. Tummers, E. H. van Veen, D. J. E. M. Roekaerts, Combust. Flame 158 (2011) 1553-1563.

[30] A. Buschmann, F. Dinkelacker, T. Schäfer, J. Wolfrum, Proc. Combust. Inst. (1996) 437-445.

[31] Y. C. Chen, N. Peters, G. A. Schneemann, N. Wruck, U. Renz, M. S. Mansour, Combust. Flame 107 (1996) 223-244.

[32] C. Duwig, L. Fuchs, P. Griebel, P. Siewert, E. Boschek, AIAA Journal 45 (2007) 624-639.

[33] S. Pfadler, A. Leipertz, F. Dinkelacker, Combust. Flame 152 (2008) 616631.

[34] N. Peters, Turbulent combustion, Cambridge University Press, Cambridge, UK, 2000. 
[35] N. Swaminathan, R. W. Bilger, B. Cuenot, Combust. Flame 126 (2001) 1764-1779.

[36] A. Trouvé, T. Poinsot, J. Fluid Mech. 278 (1994) 1-31.

[37] A. Y. Poludnenko, E. S. Oran, Combust. Flame 157 (2010) 995-1011.

[38] A. Y. Poludnenko, E. S. Oran, Combust. Flame 158 (2011) 301-326.

[39] H. Minkowski, Math. Ann. 57 (1903) 447-495.

[40] K. R. Mecke, T. Buchert, H. Wagner, Astron. Astrophys. 288 (1994) 697704.

[41] M. Kerscher, J. Schmalzing, J. Retzlaff, S. Borgani, T. Buchert, S. Gottlöber, V. Müller, M. Plionis, H. Wagner, Mon. Not. R. Astron. Soc. 284 (1997) 7384.

[42] J. Schmalzing, T. Buchert, Astrophys. J. 482 (1997) L1-L4.

[43] V. Sahni, B. S. Sathyaprakash, S. F. Shandarin, The Astrophysical Journal 495 (1998) L1-L5.

[44] J. Schmalzing, T. Buchert, A. L. Melott, V. Sahni, B. S. Sathyaprakash, S. F. Shandarin, The Astrophysical Journal 526 (1999) 568-578.

[45] J. V. Sheth, S. F. Shandarin, B. S. Sathyaprakash, Mon. Not. R. Astron. Soc. 343 (2003) 22-46.

[46] S. F. Shandarin, J. V. Sheth, V. Sahni, Mon. Not. R. Astron. Soc. 353 (2004) $162-178$. 
[47] J. V. Sheth, V. Sahni, Current Science 88 (2005) 1101-1116.

[48] M. Einasto, E. Saar, L. J. Liivamägi, J. Einasto, E. Tago, V. J. Martínez, J. L. Starck, V. Müller, P. Heínämäki, P. Nurmi, M. Gramann, G. Hütsi, Astron. Astrophys. 476 (2007) 697-711.

[49] S. L. Wilkin, C. F. Barenghi, A. Shukurov, Phys. Rev. Lett. 99 (2007) 134501.

[50] T. Leung, N. Swaminathan, P. A. Davidson, J. Fluid Mech. 710 (2012) 453481.

[51] C. N. Markides, E. Mastorakos, Proc. Combust. Inst. 30 (2005) 883-891.

[52] A. Parente, C. Galletti, L. Tognotti, Proc. Combust. Inst. 33 (2011) 33433350.

[53] M. de Joannon, A. Matarazzo, P. Sabia, A. Cavaliere, Proc. Combust. Inst. 31 (2007) 3409-3416.

[54] J. H. Chen, E. R. Hawkes, R. Sankaran, S. D. Mason, H. G. Im, Combust. Flame 145 (2006) 128-144.

[55] T. F. Lu, C. S. Yoo, J. H. Chen, C. K. Law, J. Fluid Mech. 652 (2010) 45-64.

[56] R. Borghi, D. Dutoya, Proc. Combust. Inst. 17 (1979) 235-244.

[57] K. N. C. Bray, Proc. Combust. Inst. 17 (1979) 223-233.

[58] N. Swaminathan, K. N. C. Bray, Combust. Flame 143 (2005) 549-565. 
[59] N. Swaminathan, K. N. C. Bray, Turbulent premixed flames, Cambridge University Press, Cambridge, UK, 2011.

[60] E. Mastorakos, T. A. Baritaud, T. J. Poinsot, Combust. Flame 109 (1997) $198-223$.

[61] R. Hilbert, D. Thévenin, Combust. Flame 128 (2002) 22-37.

[62] X. L. Zheng, J. Yuan, C. K. Law, Proc. Combust. Inst. 30 (2004) 415-421. 